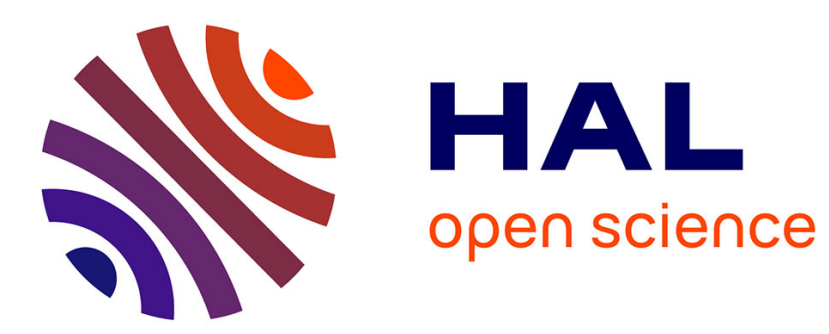

\title{
Body-fitted topology optimization of 2D and 3D fluid-to-fluid heat exchangers
}

Florian Feppon, Grégoire Allaire, Charles Dapogny, Pierre Jolivet

\section{To cite this version:}

Florian Feppon, Grégoire Allaire, Charles Dapogny, Pierre Jolivet. Body-fitted topology optimization of 2D and 3D fluid-to-fluid heat exchangers. Computer Methods in Applied Mechanics and Engineering, 2021, 376, pp.113638. 10.1016/j.cma.2020.113638 . hal-02924308v2

\section{HAL Id: hal-02924308 \\ https://hal.science/hal-02924308v2}

Submitted on 12 Nov 2020

HAL is a multi-disciplinary open access archive for the deposit and dissemination of scientific research documents, whether they are published or not. The documents may come from teaching and research institutions in France or abroad, or from public or private research centers.
L'archive ouverte pluridisciplinaire HAL, est destinée au dépôt et à la diffusion de documents scientifiques de niveau recherche, publiés ou non, émanant des établissements d'enseignement et de recherche français ou étrangers, des laboratoires publics ou privés. 


\title{
BODY-FITTED TOPOLOGY OPTIMIZATION OF 2D AND 3D FLUID-TO-FLUID HEAT EXCHANGERS
}

\author{
F. FEPPON ${ }^{12}$, G. ALLAIRE ${ }^{1}$, C. DAPOGNY ${ }^{3}$, P. JOLIVET $^{4}$ \\ ${ }^{1}$ Centre de Mathématiques Appliquées, École Polytechnique, Palaiseau, France \\ ${ }^{2}$ Safran Tech, Magny-les-Hameaux, France \\ ${ }^{3}$ Univ. Grenoble Alpes, CNRS, Grenoble INP ${ }^{1}$, LJK, 38000 Grenoble, France \\ 4 IRIT, CNRS, Toulouse, France
}

\begin{abstract}
We present a topology optimization approach for the design of fluid-to-fluid heat exchangers which rests on an explicit meshed discretization of the phases at stake, at every iteration of the optimization process. The considered physical situations involve a weak coupling between the Navier-Stokes equations for the velocity and the pressure in the fluid, and the convection-diffusion equation for the temperature field. The proposed framework combines several recent techniques from the field of shape and topology optimization, and notably a level-set based mesh evolution algorithm for tracking shapes and their deformations, an efficient optimization algorithm for constrained shape optimization problems, and a numerical method to handle a wide variety of geometric constraints such as thickness constraints and non-penetration constraints. Our strategy is applied to the optimization of various types of heat exchangers. At first, we consider a simplified 2D cross-flow model where the optimized boundary is the section of the hot fluid phase flowing in the transverse direction, which is naturally composed of multiple holes. A minimum thickness constraint is imposed on the cross-section so as to account for manufacturing and maximum pressure drop constraints. In a second part, we optimize the design of 2D and 3D heat exchangers composed of two types of fluid channels (hot and cold), which are separated by a solid body. A non-mixing constraint between the fluid components containing the hot and cold phases is enforced by prescribing a minimum distance between them. Numerical results are presented on a variety of test cases, demonstrating the efficiency of our approach in generating new, realistic, and unconventional heat exchanger designs.
\end{abstract}

Keywords. Shape and topology optimization, heat exchangers, non-mixing constraint, convective heat transfer, geometric constraints.

AMS Subject classifications. 74P10, 76B75, 35Q79.

\section{CONTENTS}

1. Introduction

2. Body-fitted topology optimization of thermal-fluid systems with null space gradient flows and geometric constraints

2.1. General setting

2.2. Shape optimization with Hadamard method

2.3. Null space gradient flows for constrained optimization

2.4. Geometric constraints based on the signed distance function and their shape derivatives

2.5. Topology optimization using a level-set based mesh evolution method

2.6. The complete algorithmic procedure

3. Topology optimization of a $2 \mathrm{D}$ air-oil heat exchanger

3.1. Setting of the study

3.2. Formulation of the shape optimization problem

3.3. Numerical results

4. Design optimization of 2D two-tubes heat exchangers involving a non-mixing constraint

4.1. Description of the physical setting

4.2. Definition of the objective functional and of the pressure drop constraint

Corresponding author. Email: florian.feppon@polytechnique.edu.

${ }^{1}$ Institute of Engineering Univ. Grenoble Alpes 
4.3. The non-mixing constraint between the fluid channels $\Omega_{f \text {,hot }}$ and $\Omega_{f \text {,cold }}$

4.4. Numerical results

5. Shape and topology optimization of a 3D fluid-to-fluid heat exchanger involving a non-mixing constraint

5.1. Formulation of the optimization problem

5.2. Selection of an initial design

5.3. Numerical solution of the topology optimization problem

5.4. Three-dimensional numerical results

\section{INTRODUCTION}

Although most of the effort of the topology optimization community has been focused on the design of mechanical structures till the 2010s, there has been recently a growing enthusiasm for multiphysics systems $[85,29,35,34,90,91,70,40]$. This is in part attributable to the recent advances in additive manufacturing techniques, which have significantly broadened the range of manufacturable designs in the aeronautic industry, including those generated by topology optimization algorithms. Another possible explanation for this observation is the development of efficient numerical techniques enabling to consider large-scale threedimensional systems, close to actual industrial interests $[1,3,19,67,13,66,56]$.

The optimization of heat exchangers is one such recent challenge, drawing more and more attention from industrial designers. These are multiphysics devices used to cool down or to heat up fluids by conveying them in the vicinity of another refrigerating or heating gas, liquid, or solid, see [55]. Industrial heat exchangers are usually composed of multiple tubes and fins shaped in order to maximize the exchange surface between the hot and cold phases. They naturally have to comply with various design constraints, such as the need for controlling the loss of pressure induced by the system on the input fluid, or the mechanical resistance of the whole device to the high thermal loads at play $[22,84,60]$.

Until recently, industrial heat exchangers have been designed with the assistance of computer-aided design (CAD) based geometry optimization software [18, 14], which are compatible with all the stages of the design workflow from physical simulations relying on commercial codes to the automated manufacturing process. Unfortunately, these methods heavily rely on the choice of a parameterization for the geometry of the optimized shape, so that they usually yield very small modifications of the initially proposed geometry $[49,23,38,36,69]$. These already allow for substantial gains in performance in most industrial applications. On the contrary, modern topology optimization algorithms are tailored to explore a much wider range of shapes, and one would naturally expect that the integration of these techniques in the engineering process could help in finding new and original designs with greater efficiency.

This motivation gave rise to a number of investigations concerned with the topology optimization for convective heat transfer involving one single fluid phase [31, 59, 64, 65, 3, 25, 29, 57, 78, 53], see also [2] for a review. A quite smaller amount of works have considered the optimal design of two-fluid heat exchangers, featuring channels conveying two types of fluids which must not interpenetrate. The topology optimization of such systems proves quite challenging, in part due to the modelling and the implementation of a non-mixing constraint between different input channels. It has been an active research issue since the seminal MSc thesis [76], see notably the recent works [80, 48, 81, 79, 87, 58] and the introduction in [54] for an overview.

Although these studies show promising results, they are all conducted in the framework of density-based topology optimization, which poses at least two major challenges. On the one hand, these methods are intrusive, that is, they may require modifications inside the numerical solver. Since they do not feature an explicit discretization of the various phases at play (solid, fluid), the physical equations describing the true "black-and-white" solid-fluid system must be replaced by approximate counterparts, defined on a fixed computational mesh in order to account for the presence of the "grayscale" transitions between different phases, which are represented by intermediate densities [20]. This delicate issue also involves the choice of interpolation parameters and suitable filters, so that a proper, "black-and-white" design be obtained at 
convergence, even though a post-processing of the final design is often required to achieve this. On the other hand, the formulation of a non-mixing constraint between different fluid phases, which is quite specific to the heat exchanger context, turns out to be particularly uneasy within density-based topology optimization frameworks: it is indeed awkward to distinguish which fluid properties should govern the approximate physics at play in the intermediate, grayscale regions. Several approaches have been proposed in the aforementioned works to circumvent this drawback, e.g.: the use of a fluid-tracking model in [76, 87], that of a suitable interpolation scheme in [58], or of a filter enforcing a solid coating around each fluid phase [48, 54]. One of the common difficulties of this class of methods is the selection of appropriate metaparameters which penalize sufficiently the porous interfaces between the two fluids but which also ensure that the inlets are indeed connected to the outlets [54].

In this article, we propose a body-fitted topology optimization methodology for heat exchangers which is based on the framework of Hadamard's method [51, 86]. When it comes to the numerical representation of designs and their updates, we do not rely on a density-based method, but rather on the level-set based mesh evolution method introduced in [5,6] and used in our previous works [42, 45]. The latter features an exact mesh of the interface between the solid material and the fluid component(s) at every iteration of the optimization process. This interface is iteratively updated according to a suitable deformation field. This strategy combines the versatility of the level-set method [72] which allows to account for complex topological changes [11, 89], with the benefits of a body-fitted approach, which keeps a neat, meshed representation of the geometry of the fluid and solid subdomains. As a result, our framework does not require any modification of the original equations describing the physical phenomena at play. Hence, any black-box software could in principle be used to achieve their numerical solution. Furthermore, all the needed geometric information about the various phases at play, such as their volume, thickness, or perimeter, are readily available from their meshed representations. In our applications to heat exchangers, this makes it possible to formulate and enforce accurately the non-mixing constraint between two fluid phases, which, as we shall see below, amounts to prescribe a minimum distance between the two fluid phases. We believe that this feature of our approach, whereby the fluid and solid subdomains are meshed explicitly at every iteration of the optimization process is one of the main advantages of our algorithm.

The other key ingredients of our numerical framework borrow from the material developed in our previous works concerned with topology optimization of multiphysics systems, involving conjugate heat transfer. The shape derivatives of quite arbitrary functionals of the domain are computed thanks to the mathematical formulas established in [42]. The considered inequality and equality constrained shape optimization problems are solved with the null space algorithm proposed and analyzed in [43]. Our three-dimensional implementation involves the solution of moderately large-scale finite element systems, computational meshes considered feature 1 to 2 million tetrahedra, which are performed thanks to domain decomposition and preconditioning techniques described in [45]. Finally, the variational method of [44] allows us to enforce conveniently our non-penetration and minimum thickness constraints.

The above numerical strategy is applied on two different heat exchanger models:

(i) our first case study is introduced in section 3: we optimize the shape of the cross-sections of oil pipes cooled down by air. We rely for this purpose on a simplified 2D physical model featuring a thermostatic boundary condition about the temperature of the optimized interface. The oil pipes are assumed to flow transversally to the air flow, which is very convenient to reduce the original $3 \mathrm{D}$ problem to a two-dimensional one. As we shall see, since no pressure drop constraint is enforced on the oil phase, the optimization problem favors the apparition of very thin and elongated structures. Therefore, a minimum thickness constraint is added to the formulation of the problem, so that the optimized designs be at the same time more relevant from a physical viewpoint and easier to fabricate. A variety of shapes are computed for various sets of physical parameters and initial shapes;

(ii) the next two test cases are concerned with the optimization of respectively 2D and 3D two-tube heat exchangers. The $2 \mathrm{D}$ test case is detailed in section 4 . We consider a fluid-to-fluid heat exchanger, where the two fluid channels, separated by a solid phase, should exchange their heat as efficiently as possible without interpenetrating. This raises the need to add a non-mixing constraint between both fluid components, or alternatively, a constraint whereby the minimum distance between them be positive. It is formulated as an averaged constraint functional involving the signed distance functions to both 
fluid subdomains, whose shape derivatives are efficiently evaluated thanks to the variational method of [44]. Let us emphasize that the implementation of this variational method is rather straightforward in both $2 \mathrm{D}$ and $3 \mathrm{D}$. This allows to circumvent the need for computing integrals along the normal rays to the optimized interface and its principal curvatures involved in the classic expression of the shape derivatives of geometric constraints, a task which is notoriously delicate to implement $[10,4,62,61]$. Our numerical results show that our method allows to enforce a wall with minimum thickness as low as a few mesh elements between two phases.

Finally, a fully three-dimensional test case is treated in section 5, where we consider the topology optimization of a two-tube heat exchanger involving the non-mixing condition. Although the physical setting and the mathematical methodology are identical to those of the previous $2 \mathrm{D}$ test case, the implementation is much more involved. In order to achieve affordable computational times, several non-trivial adaptations are indeed necessary to make the crucial stages of the framework parallel.

The remainder of this article is organized as follows. In section 2, we overview the main numerical ingredients of the proposed shape and topology optimization framework. We then turn in section 3 to the study of our first concrete case, namely the optimization of heat exchangers made of a collection of oil tubes, whose two-dimensional cross-section is the optimized variable. In section 4, we consider the optimal design of heat exchangers made of two categories of pipes, one containing a hot fluid and the other containing a cold one, in two space dimensions. We then turn in section 5 to the three-dimensional counterpart situation, where we focus on the main implementation differences with the former $2 \mathrm{D}$ case. The article ends with a series of concluding remarks and leads for future research in section 6.

\section{BODY-FITTED TOPOLOGY OPTIMIZATION OF THERMAL-FLUID SYSTEMS WITH NULL SPACE GRADIENT FLOWS AND GEOMETRIC CONSTRAINTS}

In this section, we briefly present the main ingredients of our numerical framework for the optimal design of heat exchangers, referring to the $\mathrm{PhD}$ thesis [40] and the references therein for further details. Each of the three situations tackled in sections 3 to 5 has its own distinctive particularities that shall be described in due time, but the common, abstract physical setting of this article is presented in section 2.1. The notion of Hadamard's shape derivative is recalled in section 2.2. The null space algorithm used to solve our constrained optimization problems is depicted in section 2.3. The variational method dedicated to the numerical computation of the shape derivatives of geometric constraint functionals is reviewed in section 2.4. The principles of the level-set based mesh evolution method which makes it possible to update body-fitted meshes while handling topological changes are recalled in section 2.5. Eventually, our complete algorithmic procedure is summarized in section 2.6.

\subsection{General setting}

As we have mentioned, we shall consider different physical configurations throughout this article. We introduce the common notation in the present section, referring to sections 3.1, 4.1 and 5 for further details about each individual situation.

Let $D=\overline{\Omega_{f}} \cup \overline{\Omega_{s}}$ be a "hold-all" domain, composed of two disjoint subdomains $\Omega_{f}$ and $\Omega_{s}$, separated by the interface $\Gamma=\overline{\Omega_{f}} \cap \overline{\Omega_{s}}$, as in Figure 1. For commodity, we refer to $\Omega_{f}$ as the "fluid subdomain", and to $\Omega_{s}$ as the "solid subdomain", although in the particular instance of section $3, \Omega_{s}$ is also filled with a fluid.

The velocity and pressure describing the motion of the fluid in $\Omega_{f}$ are denoted by $\boldsymbol{v}$ and $p$, respectively. These are characterized as the solutions to the incompressible steady-state Navier-Stokes equations. The temperature field $T$ inside $D$ is then influenced by convection effects according to the velocity $\boldsymbol{v}$ in the fluid domain $\Omega_{f}$, and by diffusion in the whole domain $D$. Again, see sections 3.1 and 4.1 for the precise statement of the corresponding equations. In all the numerical examples of this work, the state and adjoint equations characterizing $\boldsymbol{v}, p$ and $T$ are solved by using the finite element method. In our practical implementation, these operations as well as all other finite element based computations (such as those of shape derivatives) are achieved using the open-source FreeFEM environment [50]. The reader is referred to [45] for more details on the implementation.

We aim to optimize the geometry of the partition of $D$ into the fluid and solid phases $\Omega_{f}$ and $\Omega_{s}$. This leads us to consider generic minimization problems, that we formulate without loss of generality in terms of 


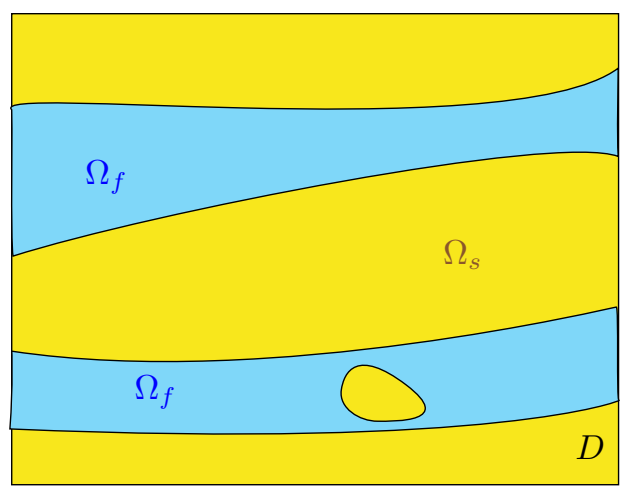

FiguRE 1. Schematic and notations for the fluid-thermal systems $D=\bar{\Omega}_{s} \cup \bar{\Omega}_{f}$ considered in section 2.1.

the fluid phase $\Omega_{f}$ :

$$
\begin{array}{cl}
\min _{\Omega_{f} \subset D} & J\left(\Omega_{f}, \boldsymbol{v}\left(\Omega_{f}\right), p\left(\Omega_{f}\right), T\left(\Omega_{f}\right)\right) \\
\text { s.t. } & \left\{\begin{array}{l}
g_{i}\left(\Omega_{f}, \boldsymbol{v}\left(\Omega_{f}\right), p\left(\Omega_{f}\right), T\left(\Omega_{f}\right)\right)=0 \text { for } 1 \leq i \leq k \\
h_{j}\left(\Omega_{f}, \boldsymbol{v}\left(\Omega_{f}\right), p\left(\Omega_{f}\right), T\left(\Omega_{f}\right)\right) \leq 0 \text { for } 1 \leq j \leq l,
\end{array}\right.
\end{array}
$$

where $J,\left(g_{i}\right)_{1 \leq i \leq k}$, and $\left(h_{j}\right)_{1 \leq j \leq l}$ are respectively the objective function, $k$ equality constraints, and $l$ inequality constraints.

\subsection{Shape optimization with Hadamard method}

Similarly to most optimization algorithms, our numerical solution of (2.1) is based on the knowledge of the derivatives of the objective and constraint functionals with respect to the optimization variable: the geometry of the fluid domain $\Omega_{f}$ in the present case. This calls for a notion of differentiation with respect to the domain, for which we rely on Hadamard's boundary variation method $[71,51,86]$.

In a nutshell, variations of $\Omega_{f}$ are considered in the form of

$$
(\operatorname{Id}+\boldsymbol{\theta})\left(\Omega_{f}\right) \text {, where } \boldsymbol{\theta} \in W^{1, \infty}\left(D, \mathbb{R}^{d}\right),\|\boldsymbol{\theta}\|_{W^{1, \infty}\left(D, \mathbb{R}^{d}\right)}<1 .
$$

The shape derivative of a functional $\Omega_{f} \mapsto F\left(\Omega_{f}\right)$ is then defined as the Fréchet derivative $\mathrm{D} F\left(\Omega_{f}\right)$ : $W^{1, \infty}\left(D, \mathbb{R}^{d}\right) \rightarrow \mathbb{R}$ of the mapping $\boldsymbol{\theta} \mapsto F\left((\operatorname{Id}+\boldsymbol{\theta})\left(\Omega_{f}\right)\right)$ at $\boldsymbol{\theta}=0$, so that the following first-order expansion holds:

$$
F\left((\mathrm{Id}+\boldsymbol{\theta})\left(\Omega_{f}\right)\right)=F\left(\Omega_{f}\right)+\mathrm{D} F\left(\Omega_{f}\right)(\boldsymbol{\theta})+\mathrm{o}(\boldsymbol{\theta}), \text { where } \frac{|\mathrm{o}(\boldsymbol{\theta})|}{\|\boldsymbol{\theta}\|_{W^{1, \infty}\left(D, \mathbb{R}^{d}\right)}} \stackrel{\boldsymbol{\theta} \rightarrow 0}{\longrightarrow} 0 .
$$

In practice, one often uses more regular vector fields $\boldsymbol{\theta}$ in (2.2) and (2.3), which additionally satisfy $\boldsymbol{\theta} \cdot \boldsymbol{n}=0$ on $\partial D$, so that $D$ is unaltered by the mapping $(\operatorname{Id}+\boldsymbol{\theta})$. Furthermore, the shape derivative $\mathrm{D} F\left(\Omega_{f}\right)(\boldsymbol{\theta})$ is often identified to an associated shape gradient $\boldsymbol{\theta}_{F}$, that is, a vector field satisfying:

$$
\forall \boldsymbol{\xi} \in V, a\left(\boldsymbol{\theta}_{F}, \boldsymbol{\xi}\right)=\mathrm{D} F\left(\Omega_{f}\right)(\boldsymbol{\xi}),
$$

where $V$ is a Hilbert space of vector fields over $D$, and $a(\cdot, \cdot)$ is an associated inner product. A usual choice is the use of a $H^{1}$ regularization,

$$
V=H^{1}(D)^{d}, \text { with } a(\boldsymbol{\theta}, \boldsymbol{\xi}):=\int_{D}(\boldsymbol{\theta} \cdot \boldsymbol{\xi}+\nabla \boldsymbol{\theta}: \nabla \boldsymbol{\xi}) \mathrm{d} x,
$$

leading to a system (2.4) and (2.5) which is easily solved by the finite element method. We refer to [21,30] for further details about this identification and regularization process of shape derivatives, and to the survey [7] for a comprehensive presentation.

In the physical context of the fluid-thermal systems described in section 2.1, the shape derivative of an arbitrary functional $F\left(\Omega_{f}\right)$, involving the solutions $(\boldsymbol{v}, p)$ and $T$ of the coupled Navier-Stokes and convectiondiffusion equations, has been calculated in [42]. We rely on the formulas provided by the Propositions 3 and 4 therein. 
Finally, let us mention some important facts about the structure of shape derivatives. Common computations yield shape derivatives $\boldsymbol{\theta} \mapsto \mathrm{D} F\left(\Omega_{f}\right)(\boldsymbol{\theta})$ written in the "volume" form of an integral on $D$, depending on $\boldsymbol{\theta}$ and $\nabla \boldsymbol{\theta}$, e.g.:

$$
\mathrm{D} F\left(\Omega_{f}\right)(\boldsymbol{\theta})=\int_{D}\left(\boldsymbol{\theta} \cdot \boldsymbol{S}\left(\Omega_{f}\right)+\nabla \boldsymbol{\theta}: T\left(\Omega_{f}\right)\right) \mathrm{d} x
$$

where the vector and matrix fields $\boldsymbol{S}\left(\Omega_{f}\right): D \rightarrow \mathbb{R}^{d}$ and $T\left(\Omega_{f}\right): D \rightarrow \mathbb{R}^{d \times d}$ depend on $\boldsymbol{v}, p, T$, and on the functional $F\left(\Omega_{f}\right)$ via suitable adjoint states. Under mild regularity assumptions, it admits the equivalent "surface" expression:

$$
\mathrm{D} F\left(\Omega_{f}\right)(\boldsymbol{\theta})=\int_{\Gamma} v_{F}\left(\Omega_{f}\right) \boldsymbol{\theta} \cdot \boldsymbol{n} \mathrm{d} s
$$

where $\boldsymbol{n}$ is the normal vector to the interface $\Gamma$, pointing outward $\Omega_{f}$, and $v_{F}\left(\Omega_{f}\right): \Gamma \rightarrow \mathbb{R}$.

In the implementation of the present work, we exclusively rely on the volume form of the shape derivative, which proves to be more robust numerically. We refer to $[52,47]$ for discussions and comparisons on this matter. The existence of the surface form, however, plays a particular role in the variational method we use to handle geometric constraint, see section 2.4 below.

\subsection{Null space gradient flows for constrained optimization}

The constrained optimization problem (2.1) is solved with the help of the null space gradient flow algorithm introduced in our previous work [43], see also Section 3.1 in [45] for a brief introduction.

Briefly, this algorithm solves nonlinear constrained optimization problems featuring a moderate number of equality and inequality constraints. In the context of a shape optimization problem such as (2.1), it calculates at each iteration $n=0,1,2, \ldots$ a deformation direction $\boldsymbol{\theta}^{n}$ of the form

$$
\boldsymbol{\theta}^{n}=-\left(\alpha_{J}^{n} \boldsymbol{\theta}_{J}^{n}+\sum_{i=1}^{k} \lambda_{i}^{n} \boldsymbol{\theta}_{g_{i}}^{n}+\sum_{j=1}^{l} \mu_{j}^{n} \boldsymbol{\theta}_{h_{j}}^{n}\right) .
$$

The vector field $\boldsymbol{\theta}_{J}^{n}$ (resp. $\boldsymbol{\theta}_{g_{i}}^{n}$ and $\boldsymbol{\theta}_{h_{j}}^{n}$ ) is a shape gradient associated to the shape derivative $\mathrm{D} J\left(\Omega_{f}^{n}\right)(\boldsymbol{\theta})$ (resp. $\mathrm{D} g_{i}\left(\Omega_{f}^{n}\right)(\boldsymbol{\theta})$ and $\left.\mathrm{D} h_{j}\left(\Omega_{f}^{n}\right)(\boldsymbol{\theta})\right)$ via (2.4). The coefficients $\alpha_{J}^{n}, \lambda_{i}^{n}$, and $\mu_{j}^{n}$ are automatically computed as the solution to a dual quadratic optimization subproblem which allows to identify the subset of inequality constraints which must remain saturated (or "active", that is meeting their respective upper bound) in the course of the optimization process. This yields an "optimal" descent displacement field $\boldsymbol{\theta}^{n}$, in the sense that update steps $\left(\mathrm{Id}+\boldsymbol{\theta}^{n}\right)\left(\Omega_{f}\right)$ tend to decrease the objective function $J$ while reducing gradually the violation of the constraints $g_{i}$ and $h_{j}$.

\subsection{Geometric constraints based on the signed distance function and their shape derivatives}

As advocated in [68, 10], a wide variety of geometric constraints on a shape $\Omega \subset D$ can be formulated as averaged criteria depending on the signed distance function $d_{\Omega}$ to $\Omega$. As such, minimum and maximum thickness constraints were considered in this way in $[68,10]$ and we shall rely on a similar approach in section 3.2.2. Moreover, we shall express the non-mixing constraint between the hot and cold phases of a two-fluid heat exchanger in this framework in section 4.3.

In this section, $\Omega \subset \subset D$ is a smooth shape, which stands for either the whole fluid domain $\Omega_{f}$ in the context of section 3, or for either the hot or the cold fluid component $\Omega_{f \text {, hot }}$ or $\Omega_{f \text {,cold }} \subset \Omega_{f}$ in the configuration of two-fluid heat exchangers considered in sections 4 and 5.

The signed distance function $d_{\Omega}$ to $\Omega$ is defined for all $x \in D$ by

$$
d_{\Omega}(x)=\left\{\begin{aligned}
-d(x, \partial \Omega) & \text { if } x \in \Omega \\
0 & \text { if } x \in \partial \Omega \\
d(x, \partial \Omega) & \text { if } x \in D \backslash \bar{\Omega},
\end{aligned}\right.
$$

where $d(x, \partial \Omega)$ is the Euclidean distance from the point $x$ to the boundary $\partial \Omega$ :

$$
d(x, \partial \Omega):=\inf _{p \in \partial \Omega}|x-p| .
$$

Let us recall a few useful notions and facts related to the signed distance function, see also Figure 2: 


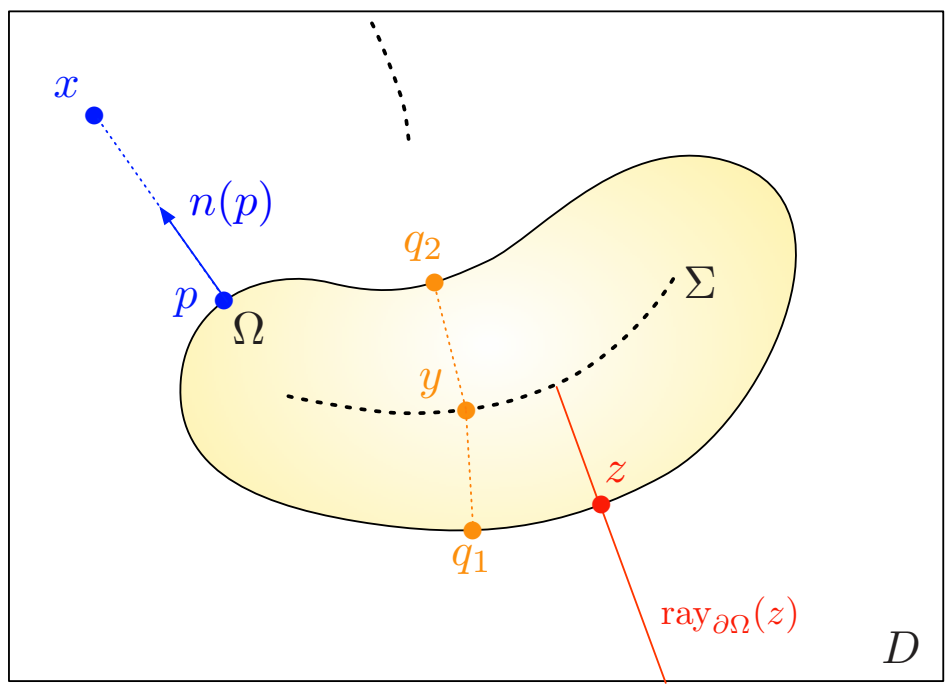

FiguRE 2. Illustration of the main objects attached to the signed distance function. The point $x \in D \backslash \Sigma$ has $p \in \partial \Omega$ as unique projection point, while $y \in \Sigma$ has $q_{1}$ and $q_{2}$ for projections onto $\partial \Omega$.

(i) the set of points $p$ where the infimum is realized in (2.8) is the set of projections of $x$ onto $\partial \Omega$. When there exists a unique such point, it is called the projection of $x$ onto $\partial \Omega$, and it is denoted by $p_{\partial \Omega}(x)$;

(ii) the set of points $x \in D$ having at least two distinct projections onto $\partial \Omega$ is called the skeleton (or sometimes, the medial axis) $\Sigma$ of $\Omega$. It is a set with null Lebesgue measure, and since $\Omega$ is smooth, so is the closure $\bar{\Sigma}$, see [33, 63];

(iii) the set of points $x \in D \backslash \Sigma$ sharing the same projection point $z \in \partial \Omega$ is called the normal ray of $z$ :

$$
\operatorname{ray}_{\partial \Omega}(z)=\left\{x \in D \backslash \Sigma, p_{\partial \Omega}(x)=z\right\} ;
$$

(iv) the signed distance function is differentiable at every point $x \in D \backslash \Sigma$, and its derivative reads:

$$
\nabla d_{\Omega}(x)=\boldsymbol{n}\left(p_{\partial \Omega}(x)\right) .
$$

The signed distance function $d_{\Omega}$ conveniently encodes most of the geometric features of the domain $\Omega$. We refer to [33] for an extensive discussion of this notion, and to Section 1.3 in [40] for an introduction. The geometric constraints of interest in this work assume the form:

$$
P(\Omega) \leq 0 \text { where } P(\Omega):=\int_{D} j\left(d_{\Omega}(x)\right) \mathrm{d} x,
$$

for a given smooth function $j: \mathbb{R} \rightarrow \mathbb{R}$; see sections 3.2 .2 and 4.3 for several instances of such geometric constraints.

As we have seen in sections 2.2 and 2.3, the solution of the optimization program (2.1) relies on the shape derivative $\mathrm{D} P(\Omega)$ of $P$. The numerical computation of the shape derivative of functions $P(\Omega)$ of the form (2.10), depending on the signed distance function $d_{\Omega}$, requires a special treatment which we now summarize, referring to $[4,68,10]$ for details. The shape derivative of $P(\Omega)$ reads:

$$
\mathrm{D} P(\Omega)(\boldsymbol{\theta})=\int_{D} j^{\prime}\left(d_{\Omega}(x)\right) d_{\Omega}^{\prime}(\boldsymbol{\theta})(x) \mathrm{d} x .
$$

In the above expression, $d_{\Omega}^{\prime}(\boldsymbol{\theta})$ is the "Eulerian derivative" of the signed distance function in the direction $\boldsymbol{\theta} \in W^{1, \infty}\left(D, \mathbb{R}^{d}\right)$, that is, for a.e. $x \in D, d_{\Omega}^{\prime}(\boldsymbol{\theta})(x)$ is the Fréchet derivative of the mapping $\boldsymbol{\theta} \mapsto d_{\Omega}(\boldsymbol{\theta})(x)$ at $\boldsymbol{\theta}=0$. This function satisfies the following boundary-value problem:

$$
\left\{\begin{array}{rlrl}
\nabla d_{\Omega}^{\prime}(\boldsymbol{\theta}) \cdot \nabla d_{\Omega} & =0 & & \text { in } D \backslash \bar{\Sigma} \\
d_{\Omega}^{\prime}(\boldsymbol{\theta}) & =-\boldsymbol{\theta} \cdot \boldsymbol{n} & & \text { on } \partial \Omega, \\
7 &
\end{array}\right.
$$


where $\boldsymbol{n}$ denotes the normal vector to $\partial \Omega$, pointing outward $\Omega$. The first line of (2.12) expresses that the values of $d_{\Omega}^{\prime}(\boldsymbol{\theta})$ are constant along the rays (2.9) of $\partial \Omega$, while the second line actually specifies these values on $\partial \Omega$.

The expression (2.11) is uneasy to handle in practice, since the derivative $d_{\Omega}^{\prime}(\boldsymbol{\theta})$ depends in a non-trivial way on $\boldsymbol{\theta}$. Owing to the coarea formula, (2.11) can be rewritten in the following surface form:

$$
\mathrm{D} P(\Omega)(\boldsymbol{\theta})=\int_{\partial \Omega} v_{P}(\Omega) \boldsymbol{\theta} \cdot \boldsymbol{n} \mathrm{d} s
$$

see $[4,10,44]$. Unfortunately, the analytical expression of the scalar field $v_{P}(\Omega): \Gamma \rightarrow \mathbb{R}$ (which is not reported here for brevity) involves integrals along the normal rays to $\partial \Omega$, as well as the principal curvatures of $\partial \Omega$. These quantities are well-known to be difficult to compute in a robust way on unstructured meshes.

These issues can be overcome thanks to the variational method from our previous work [44]: instead of evaluating the analytic formula for the function $v_{P}(\Omega)$, we actually compute it numerically as the solution to a variational problem. More precisely, we consider the problem

find $v \in V_{\omega}$ such that for any $w \in V_{\omega}$,

$$
\int_{\partial \Omega} v w \mathrm{~d} s+\int_{D \backslash \bar{\Sigma}} \omega\left(\nabla d_{\Omega} \cdot \nabla v\right)\left(\nabla d_{\Omega} \cdot \nabla w\right) \mathrm{d} x=-\int_{D} j^{\prime}\left(d_{\Omega}\right) w \mathrm{~d} x
$$

which is easily solved numerically by means of standard piecewise linear finite elements. In the above formulation, the function $\omega$ is a positive weight which is continuous on $D \backslash \bar{\Sigma}$, while $V_{\omega}$ is a weighted Hilbert space, whose precise definition and mathematical properties are described in Section 2 of [44].

The space $V_{\omega}$ contains functions $w$ which are constant along the sets $\operatorname{ray}_{\partial \Omega}(z), z \in \partial \Omega$, and possibly discontinuous across $\bar{\Sigma}$. Recalling (2.12), one may then insert $d_{\Omega}^{\prime}(\boldsymbol{\theta})$ as test function in (2.14), and it follows from the expression (2.12) that the trace of the solution $v \in V_{\omega}$ to (2.14) on $\Gamma$ is exactly the desired scalar field $v_{P}\left(\Omega_{f}\right)$.

The weight $\omega$ can be chosen rather arbitrarily. In theory, from [44], it should only be continuous and positive on $D \backslash \bar{\Sigma}$ and satisfy a few additional monotonicity assumptions. It is however numerically desirable that $\omega$ vanish on the skeleton $\bar{\Sigma}$ of $\Omega$ so that the discontinuities of test functions $w \in V_{\omega}$ across $\Sigma$ are better captured with piecewise linear finite elements, thus enhancing the accuracy of the calculation of $v_{P}\left(\Omega_{f}\right)$. In all the situations addressed in this work, following the recommendation in [44] (section 3.3.1) we use the weight

$$
\omega=\frac{2}{1+100\left|d_{\Omega} \Delta d_{\Omega}\right|^{3.5}}
$$

a choice which is motivated by the large values of the discretized version of $\Delta d_{\Omega}$ near $\bar{\Sigma}$. The constants 100 or 3.5 have been selected empirically to achieve universally satisfactory performance even when the distance of $\partial \Omega$ to the skeleton $\bar{\Sigma}$ is small. In most cases, the use of different constants works equally well.

\subsection{Topology optimization using a level-set based mesh evolution method}

Among the multiple available approaches, see [7] for an overview, we rely on the level-set based mesh evolution algorithm from our previous works $[5,6,42,45]$ for the numerical representation of shapes and their deformations, see also [77] for earlier related ideas. The salient feature of this approach is that it enjoys an explicit, meshed representation of the tracked subdomains $\Omega_{f}$ and $\Omega_{s}$ at any stage of the optimization process, while still allowing for topological changes. This is possible thanks to the combination of two complementary numerical representations of the partition $D=\overline{\Omega_{f}} \cup \overline{\Omega_{s}}$ :

(i) a meshed description: a conforming simplicial mesh $\mathcal{T}$, i.e., composed of triangles in $2 \mathrm{D}$ and tetrahedra in $3 \mathrm{D}$, of the total domain $D$ is available, in which the interface $\Gamma$ is meshed explicitly. This representation is well-suited when it comes to solving the physical state equations with the finite element method on their associated meshed subdomains $\Omega_{f}$ and $\Omega_{s}$, see, e.g., Figs. 5, 12 and 23d in the results below;

(ii) a level-set description: following the pioneering work [73], see also [82] for an overview and [12, 89, 83, 74] for the seminal applications in shape and topology optimization, $\Omega_{f}$ and $\Omega_{s}$ are represented by means 
of a scalar function $\phi: D \rightarrow \mathbb{R}$, in practice discretized at the vertices of a mesh of $D$, such that:

$$
\begin{cases}\phi(x)<0 & \text { if } x \in \Omega_{f} \\ \phi(x)=0 & \text { if } x \in \Gamma \\ \phi(x)>0 & \text { if } x \in \Omega_{s} .\end{cases}
$$

This representation allows to account for large deformations of the configuration $D=\overline{\Omega_{f}} \cup \overline{\Omega_{s}}$ according to a given velocity field $\boldsymbol{\theta}$ and a pseudo-time step $\tau>0$. One solves to this end the following linearized version of the level-set advection equation:

$$
\left\{\begin{array}{cl}
\frac{\partial \psi}{\partial t}(t, x)+\boldsymbol{\theta}(x) \cdot \nabla \psi(t, x)=0 & (t, x) \in(0, \tau) \times D \\
\psi(0, x)=\phi(x) & x \in D,
\end{array}\right.
$$

which yields an updated level-set function $\psi(\tau, \cdot)$ associated to the partition $D=\overline{\left(\Omega_{f}\right)_{\tau \boldsymbol{\theta}}} \cup \overline{\left(\Omega_{s}\right)_{\tau \boldsymbol{\theta}}}$, where $\left(\Omega_{f}\right)_{\tau \boldsymbol{\theta}}$ is the negative subdomain of $\phi(\tau, \cdot)$ and $\left(\Omega_{s}\right)_{\tau \boldsymbol{\theta}}:=D \backslash \overline{\left(\Omega_{f}\right)_{\tau \boldsymbol{\theta}}}$.

Dedicated numerical algorithms allow to switch from one of these descriptions to the other. On the one hand, a level-set function $\phi$ associated to a configuration $D=\overline{\Omega_{f}} \cup \overline{\Omega_{s}}$ under meshed description is generated as the signed distance function to $\Omega_{f}$ by using the open-source software Mshdist [28]. Conversely, a conforming mesh of $D$ where both subdomains $\Omega_{f}$ and $\Omega_{s}$ are explicitly discretized is generated from the datum of an associated level-set function $\phi: D \rightarrow \mathbb{R}$ thanks to the remeshing library Mmg [27, 26], see also the aforementioned works $[5,6,42,45]$ for illustrations and details.

\subsection{The complete algorithmic procedure}

The shape and topology optimization algorithm derived from the previous considerations is summarized in algorithm 1 below. Note that we refer to our method as a "shape and topology optimization algorithm" (although we rely solely on shape derivatives) because the boundaries of the design domain are allowed to move freely and topological changes occurring in the course of the optimization are captured. Our approach is therefore substantially different from the many works concerned with parametric shape optimization of fluid systems [49, 39, 23, 38], whereby very small updates of the design shape are sought.

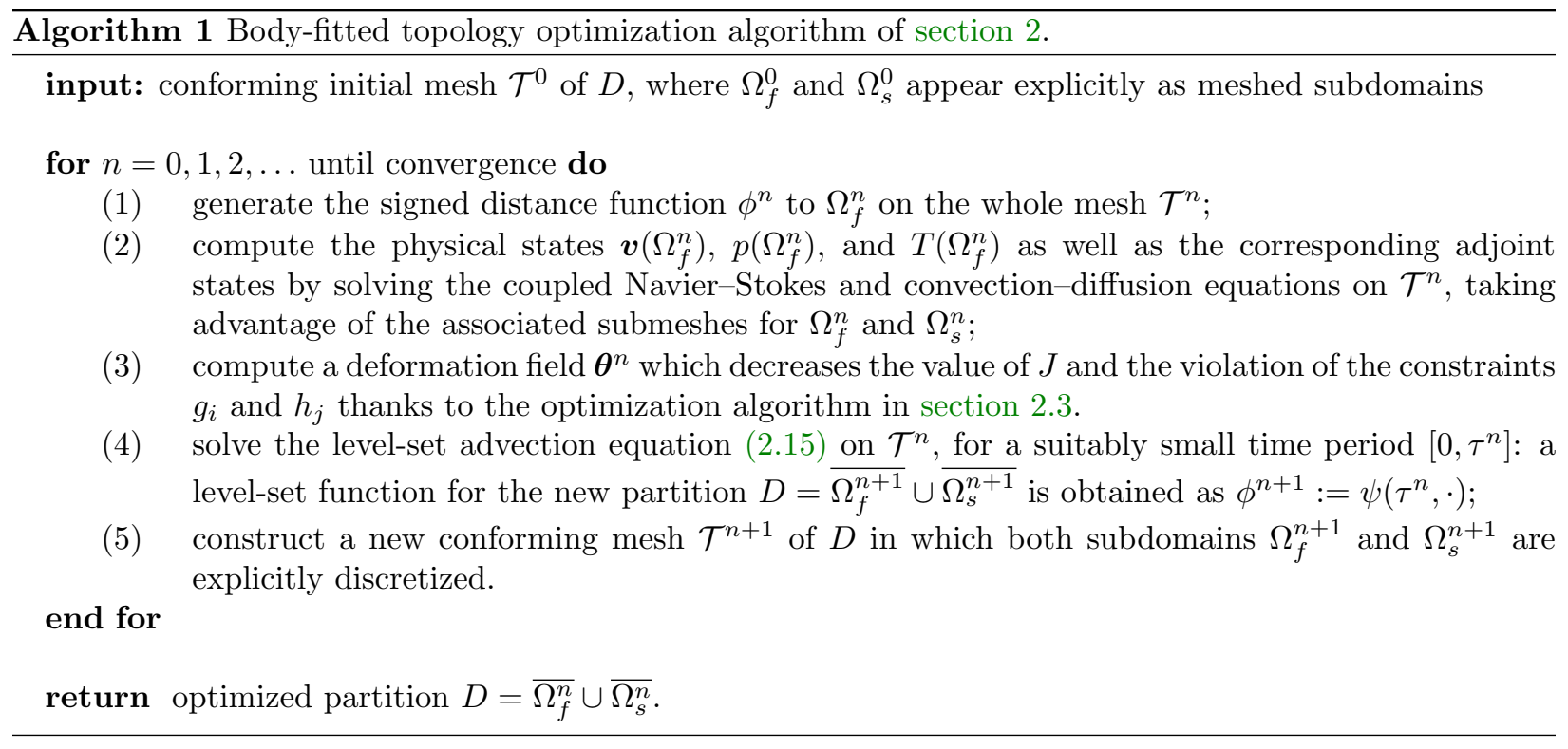




\section{Topology optimization of A 2D AIR-OIL HeAT EXChANGER}

In this section, we deal with the optimization of the $2 \mathrm{D}$ cross-section of a three-dimensional air-oil heat exchanger. The setting of this problem is described in section 3.1. The two phases $\Omega_{f}$ and $\Omega_{s}$ are filled with air and oil, respectively. The physical phenomena of interest solely take place in $\Omega_{f}$ : the velocity and pressure $(\boldsymbol{v}, p)$ of the fluid are governed by the 2D incompressible steady-state Navier-Stokes equations, and the temperature $T$ inside $\Omega_{f}$ is subject to diffusion and convection by the fluid, with a thermostatic boundary

condition on the interface $\Gamma$. The optimization problem is formulated in section 3.2, where we define the considered shape functionals, and notably a minimum thickness constraint imposed on the oil phase $\Omega_{s}$. Section 3.3 presents a variety of numerical results, obtained by applying algorithm 1 with different initial designs and sets of physical parameters.

Remark 1. Here and in the sequel, physical units are omitted when it comes to the numerical values used in our test cases. For simplicity, we prefer to work with nondimensional quantities, and we do not claim that all values are completely relevant from the physical point of view. In particular, because of computational limitations, our choices of Reynolds and Péclet numbers (which are nondimensional in the first place) are quite restricted to moderate values. Nevertheless, one can think that the physical units are SI units as follows. All lengths are understood in meters $(\mathrm{m})$, velocities in $\mathrm{ms}^{-1}$, temperatures in Kelvin (K) or degree Celsius in the next sections, fluid density in $\mathrm{kg} \mathrm{m}^{-3}$, fluid thermal capacity in $\mathrm{J} \mathrm{K}^{-1} \mathrm{~kg}^{-1}$, thermal conductivity in $\mathrm{J} \mathrm{K}^{-1} \mathrm{~m}^{-1} \mathrm{~s}^{-1}$, and fluid viscosity in $\mathrm{kg} \mathrm{m}^{-1} \mathrm{~s}^{-1}$.

\subsection{Setting of the study}

Our purpose is to optimize the shape and the topology of a $2 \mathrm{D}$ air-oil heat exchanger. The situation is that depicted on Figure 3: the hold-all domain $D=(0, L) \times(0, H)$ is a rectangle with width $L=0.85$ and height $H=1$. It contains a fluid, air, which occupies a subdomain $\Omega_{f} \subset D$ to be optimized. The complementary subdomain $\Omega_{s}=D \backslash \overline{\Omega_{f}}$ is filled with oil. The fluid boundary

$$
\partial \Omega_{f}=\overline{\partial \Omega_{f, \text { in }}} \cup \overline{\partial \Omega_{f, \text { out }}} \cup \overline{\partial \Omega_{f, \text { wall }}} \cup \bar{\Gamma},
$$

is composed of the following regions:

- $\partial \Omega_{f, \text { in }}$ is the inlet boundary, where the fluid is entering the device with a parabolic velocity $\boldsymbol{v}_{0}$ with maximum norm $\left\|\boldsymbol{v}_{0}\right\|_{\infty}$;

- $\partial \Omega_{f \text {,out }}$ is the outlet boundary, where the fluid is exiting the device with zero normal stress;

- $\partial \Omega_{f \text {,wall }}$ is the remaining part of the device $D$, which bears a no-slip boundary condition for the fluid and an adiabatic boundary condition for the temperature;

- $\Gamma=\overline{\Omega_{f}} \cap \overline{\Omega_{s}}$ is the interface between the air and oil phases. The oil inside $\Omega_{s}$ has a much higher thermal conductivity than that of air, and so the temperature takes the uniform value $T=T_{\text {oil }}$ in $\Omega_{s}$. Moreover, the interface $\Gamma$ is sufficiently thin and conductive so that $T=T_{\text {oil }}$ on $\Gamma$.

From a physical point of view, after 3D extrusion in the $z$-direction, this system can be interpreted as a heat exchanger featuring air flowing in the $x$-direction and cooling down infinitely long oil channels flowing in the transverse $z$-direction. 


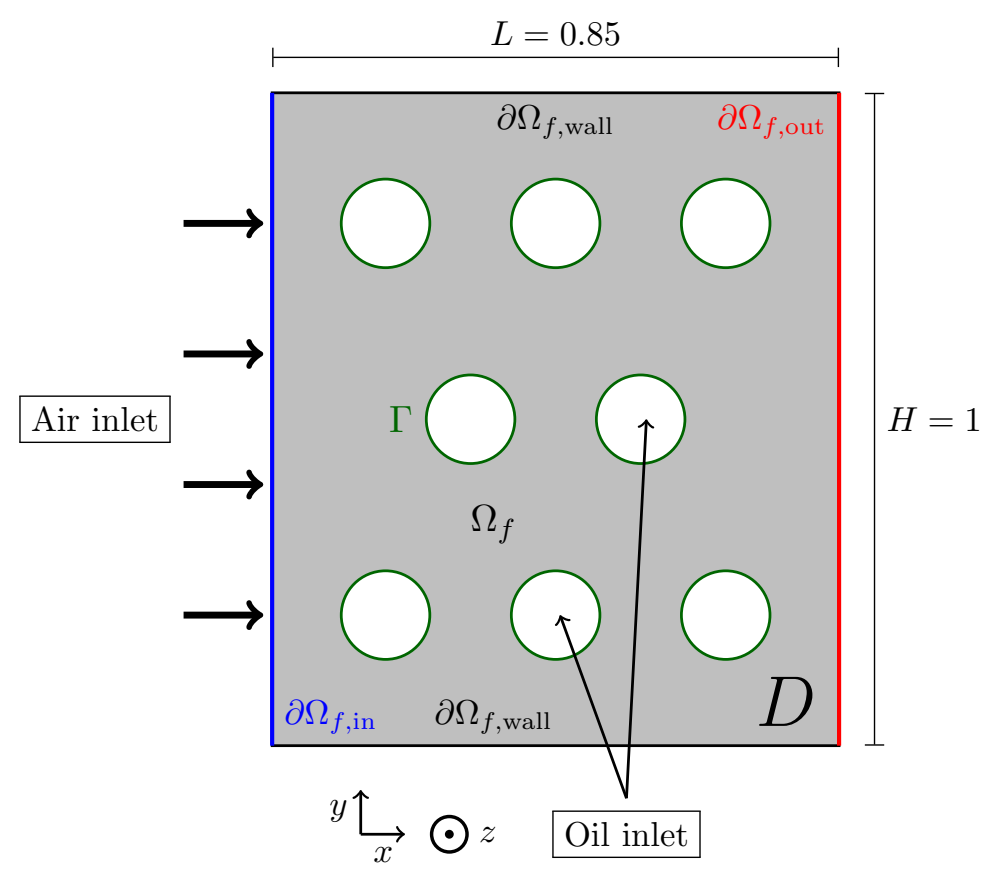

Figure 3. Setting of the air-oil heat exchanger of section 3.

Mathematically, the fluid, air, is characterized by its velocity $\boldsymbol{v}$ and pressure $p$ in $\Omega_{f}$, which are the solutions to the incompressible steady-state Navier-Stokes equations:

$$
\left\{\begin{array}{rlrl}
-\operatorname{div}\left(\sigma_{f}(\boldsymbol{v}, p)\right)+\rho \nabla \boldsymbol{v} \boldsymbol{v} & =0 & & \text { in } \Omega_{f} \\
\operatorname{div}(\boldsymbol{v}) & =0 & & \text { in } \Omega_{f} \\
\boldsymbol{v} & =\boldsymbol{v}_{0} & & \text { on } \partial \Omega_{f, \text { in }} \\
\sigma_{f}(\boldsymbol{v}, p) \boldsymbol{n} & =0 & \text { on } \partial \Omega_{f, \text { out }} \\
\boldsymbol{v} & =0 & \text { on } \partial \Omega_{f, \text { wall }} \\
\boldsymbol{v} & =0 & \text { on } \Gamma .
\end{array}\right.
$$

Here, $\rho$ is the fluid density, and the fluid stress tensor $\sigma_{f}(\boldsymbol{v}, p)$ is given by Newton's law:

$$
\sigma_{f}(\boldsymbol{v}, p)=2 \nu e(\boldsymbol{v})-p \mathbf{I},
$$

in which $e(\boldsymbol{v})=\frac{1}{2}\left(\nabla \boldsymbol{v}+\nabla \boldsymbol{v}^{T}\right)$ is the rate of strain tensor, $\nu$ is the dynamic viscosity of the fluid, and I is the identity $2 \times 2$ matrix. The temperature field $T$ inside the air phase $\Omega_{f}$ is then determined in terms of the velocity $\boldsymbol{v}$ through the following convection-diffusion equation:

$$
\left\{\begin{array}{rlrl}
-\operatorname{div}\left(k_{f} \nabla T\right)+\rho c_{p} \boldsymbol{v} \cdot \nabla T & =0 & \text { in } \Omega_{f} \\
T & =T_{\text {in }} & & \text { on } \partial \Omega_{f, \text { in }} \\
-k_{f} \frac{\partial T}{\partial \boldsymbol{n}} & =0 & \text { on } \partial \Omega_{f} \backslash \partial \Omega_{f, \text { in }} \\
T & =T_{\text {oil }} & \text { on } \Gamma,
\end{array}\right.
$$

where $k_{f}$ and $c_{p}$ are the thermal conductivity and the thermal capacity of the fluid, respectively. Let us emphasize that, thanks to the thermostatic boundary condition $T=T_{\text {oil }}$ imposed on the interface $\Gamma$, no model is needed for describing the motion of the oil phase in $\Omega_{s}$, and the coupled system of physical equations (3.1) and (3.3) is posed solely on $\Omega_{f}$.

In our application, the "cold" air flow is entering the domain $D$ on the left-hand side with a temperature $T_{\mathrm{in}}=310$. The temperature on the interface $\Gamma$ is set to $T_{\text {oil }}=400$. The density and the capacity coefficients of the fluid are $\rho=1$ and $c_{p}=1$. Since we do not rely on a turbulence model in the Navier-Stokes equations 


\begin{tabular}{|c|c|c|c|c|c|}
\hline Configuration & Input velocity $\left\|\boldsymbol{v}_{0}\right\|$ & Pressure loss threshold $\mathrm{DP}_{0}$ & $\mathrm{Re}$ & $\mathrm{Pe}$ & $\mathrm{J}_{0}$ \\
\hline$\# 1$ & 10 & 1300 & 40 & 475 & 4500 \\
\hline$\# 2$ & 25 & 1030 & 100 & 1200 & 10,000 \\
\hline$\# 3$ & 40 & 475 & 160 & 1900 & 9500 \\
\hline
\end{tabular}

TABLE 1. Configurations considered for the input velocity and pressure constraint values.

(3.1) for the determination of the fluid velocity and pressure $(\boldsymbol{v}, p)$, our study is restricted to moderate values of Reynolds and Péclet numbers Re and Pe. The viscosity and conductivity coefficients $\nu$ and $k_{f}$ are set so that the values

$$
\operatorname{Re}:=\frac{\rho\left\|\boldsymbol{v}_{0}\right\|_{\infty} H}{\nu} \quad \text { Pe }:=\frac{\rho c_{p}\left\|\boldsymbol{v}_{0}\right\|_{\infty} H}{k_{f}},
$$

are those associated to the three different configurations described in Table 1.

\subsection{Formulation of the shape optimization problem}

\subsubsection{Definition of the objective and constraint functions}

In this first physical setting, the heat exchanged between the air, $\Omega_{f}$, and the oil, $\Omega_{s}$, phases is maximized, while imposing an upper bound $\mathrm{DP}_{0}$ on the static pressure drop between the inlet and the outlet of the device. The considered shape and topology optimization problem (2.1) reads in this case:

$$
\begin{aligned}
\min _{\Omega_{f} \subset D} J\left(\Omega_{f}\right):=-\int_{\Omega_{f}} \rho c_{p} \boldsymbol{v} \cdot \nabla T \mathrm{~d} x \\
\text { s.t. } \quad \operatorname{DP}\left(\Omega_{f}\right):=\int_{\partial \Omega_{f, \text { in }}} p \mathrm{~d} s-\int_{\partial \Omega_{f, \text { out }}} p \mathrm{~d} s \leq \mathrm{DP}_{0} .
\end{aligned}
$$

Upon integration by parts, the objective function $J\left(\Omega_{f}\right)$ rewrites exactly as the difference between the heat entering from the inlet $\partial \Omega_{f, \text { in }}$ and that exiting from $\partial \Omega_{f, \text { out }}$ :

$$
J\left(\Omega_{f}\right)=-\left(\int_{\partial \Omega_{f, \text { in }}} \rho c_{p} T \boldsymbol{v} \cdot \boldsymbol{n} \mathrm{d} s+\int_{\partial \Omega_{f, \text { out }}} \rho c_{p} T \boldsymbol{v} \cdot \boldsymbol{n} \mathrm{d} s\right),
$$

where we recall that the normal vector $\boldsymbol{n}$ is pointing outward $D$. The first term in the above right-hand side is constant since $\boldsymbol{v}=\boldsymbol{v}_{0}$ on $\partial \Omega_{f, \text { in }}$, so that minimizing $J\left(\Omega_{f}\right)$ is equivalent to maximizing the heat leaving the device through $\partial \Omega_{f, \text { out }}$, which is also the heat extracted from the oil channels $\Omega_{s}$.

Remark 2. Strictly speaking, sufficient regularity is expected for the pressure $p$, which is only a priori an element of $L^{2}\left(\Omega_{f}\right)$, to have a trace on the inlet and outlet boundaries. The shape derivative of $\operatorname{DP}\left(\Omega_{f}\right)$ is computed with the formulas of [40], which requires the solution to a fluid adjoint system involving the linearized transpose of the Navier-Stokes operator and a boundary integral involving a pressure test variable as a right-hand side.

\subsubsection{Minimum thickness constraint for the oil phase cross-section $\Omega_{s}$}

As evidenced by the results of section 3.3 below, the solution of the optimization problem (3.4) tends to produce very thin and elongated patterns for the oil cross-section $\Omega_{s}$. From a physical point of view, these would induce an important pressure drop in the transverse oil channels of the underlying three-dimensional device, making the overall design impractical. Furthermore, such thin and elongated components are numerically instable. Indeed, patterns of the design smaller than the prescribed mesh size tend to disappear in the course of the remeshing steps due to smoothing. This calls for the addition of a constraint on the minimum thickness $d_{\min }$ of the oil channels $\Omega_{s}$ in the shape optimization problem (3.4), which also improves the manufacturability of the optimized device. Our modeling of this constraint follows the strategy proposed in [68]: we replace the shape and topology optimization problem (3.4) with a variant where the performance of the system $J\left(\Omega_{f}\right)$ is constrained to be at least as good as a threshold value $\mathrm{J}_{0}$. The minimized function 
$E\left(\Omega_{f}\right)$ favors areas of $D \backslash \Omega_{f}$ thicker than $d_{\min }$, and its shape derivative vanishes in those regions which are already thicker than $d_{\text {min }}$ :

$$
\begin{array}{ll}
\min _{\Omega_{f} \subset D} & E\left(\Omega_{f}\right):=-\int_{D \backslash \Omega_{f}} d_{\Omega_{f}}^{2} \max \left(-d_{\Omega_{f}}+d_{\min } / 2,0\right)^{2} \mathrm{~d} x \\
\text { s.t. } & \left\{\begin{array}{c}
\mathrm{DP}\left(\Omega_{f}\right) \leq \mathrm{DP}_{0} \\
J\left(\Omega_{f}\right) \geq \mathrm{J}_{0} .
\end{array}\right.
\end{array}
$$

In the present setting, the minimum thickness value is set to $d_{\min }:=0.027$. As already mentioned, the shape derivatives of the "mechanical" functionals $J\left(\Omega_{f}\right)$ and $\operatorname{DP}\left(\Omega_{f}\right)$, depending on $\Omega_{f}$ via the solutions $\boldsymbol{v}\left(\Omega_{f}\right)$, $p\left(\Omega_{f}\right)$, and $T\left(\Omega_{f}\right)$ to the physical equations (3.1) and (3.3), have been calculated in our previous work [42], and their expressions are omitted for brevity. The shape derivative of the functional $E\left(\Omega_{f}\right)$, of the form (2.10), is evaluated numerically with the variational method of section 2.4. The threshold $\mathrm{J}_{0}$ measuring the performance of the heat exchanger is determined empirically from the values obtained from the solution of the original problem (3.4) without the minimum thickness constraint. This value is set depending on the configuration considered according to Table 1.

\subsection{Numerical results}

We optimize the design of the considered heat exchangers without and with the minimum thickness constraint of section 3.2.2. Namely, we solve both optimization problems (3.4) and (3.5), for the three sets of parameters of Table 1. In order to further illustrate the strong dependence of optimized designs on the corresponding initializations, we propose a set of 24 exploratory results, corresponding to 4 different initial topologies and 3 sets of imposed velocities and allowed pressure drops (plus the 2 cases with or without thickness constraint). These are reported in Table 2 below where the final temperature and kinetic energy fields are also displayed. Let us note that the existence of different (local) minima which can be obtained by using as many different initializations is often seen as a drawback in mathematical optimization; the present numerical experiment shows, however, that it is rather an advantage from an engineering perspective since it leaves the room for many possible designs which can accommodate other practical constraints, not yet taken into account.

As is visible on Table 2, the maximum pressure drop constraint is not saturated in all the situations where $\left\|\boldsymbol{v}_{0}\right\|_{\infty}=10$. In test cases $\# 3$, $\# 5$, thin structures have been removed due to remeshing in the course of the optimization process. For some of the other test cases, the topology changes due to the elongation of the oil phase which creates additional connected components (e.g. in \#19 and \#20 where the number of oil components increases from 5 to 7 ). Last, in the configurations featuring the minimum thickness constraint formulated as (3.5), the prescribed threshold values $\mathrm{J}_{0}$ and $\mathrm{DP}_{0}$ are often too ambitious. As a result these constraints are slightly violated by the final design because the optimization algorithm had to find a compromise between the two. This is visible for instance on $\# 24$, where the pressure drop $\mathrm{DP}_{\text {final }}$ is much worse as in the corresponding case \#23 where the thickness constraint is not imposed, but which features also a much better heat exchanged $J_{\text {final }}$.

Since the heating of the air flow is due to a Dirichlet boundary condition on the oil channels, the optimization algorithm has a tendency to increase the length of the oil/air interface, which simultaneously increases the amount of heat exchanged between oil and air. For large velocities, the oil channels are elongated with an aerodynamic profile in order to meet the pressure drop limit. Note that the volume of the oil channels is not constrained and thus varies a lot from one case to another. In some cases, such as \#8, \#10, \#14, \#19, \#20, \#22 and \#24, the initial topology is a collection of oil "islands" and it is transformed, after optimization, into a kind of oil "matrix" perforated by air channels. This is a feature of topology optimization algorithms which cannot be achieved in practice by parametric optimization algorithms, even if the geometry of the shape can be modified by moving the mesh. The key point is here the use of a remeshing algorithm which allows for very large mesh deformations.

Remarkably, the optimization algorithm generates recirculating fluid patterns opposed to the direct flow, e.g., on cases \#8-\#12 and \#20-\#24, which increase the amount of exchanged heat by widening the surface contact area between the hot oil phase and the incoming flow. Note that [78] has also reported the benefit of recirculating patterns, such as counter-rotating vortices, when it comes to heat exchange. Furthermore, the conducted numerical procedure has selected aerodynamic designs for the transverse oil channels so as to 
limit the output pressure loss. Finally, the effect of the minimum thickness constraint in (3.5) is particularly significant on the configurations where the input velocity is maximum, $\left\|\boldsymbol{v}_{0}\right\|_{\infty}=40$, which are also those favoring the most elongated structures.

A few intermediate shapes illustrating the shape optimization process in some of these experiments are reported in Figs. 4a to 4c. Finally, all these results were produced with a rather fine mesh resolution for the bounding box $D$ : the minimum edge size was of the order of $h_{\min }=0.003$, which corresponds to meshes with approximately 30,000 vertices. The whole optimization process of a single test case involves about 400 iterations which took about 12 hours without the use of parallel computing. As an illustration the mesh of the final shape is displayed on Figure 5 for one of the 24 situations considered. 


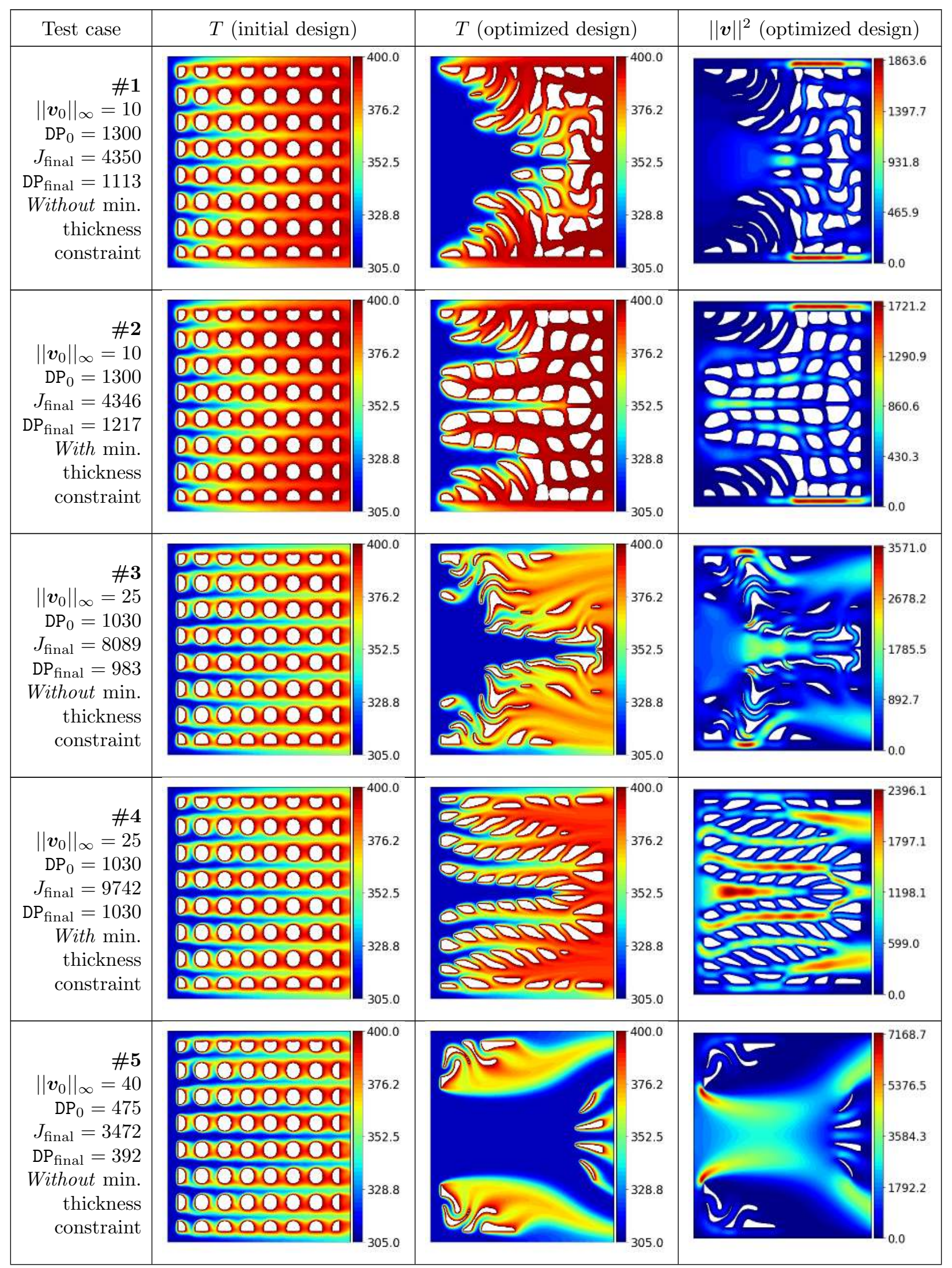




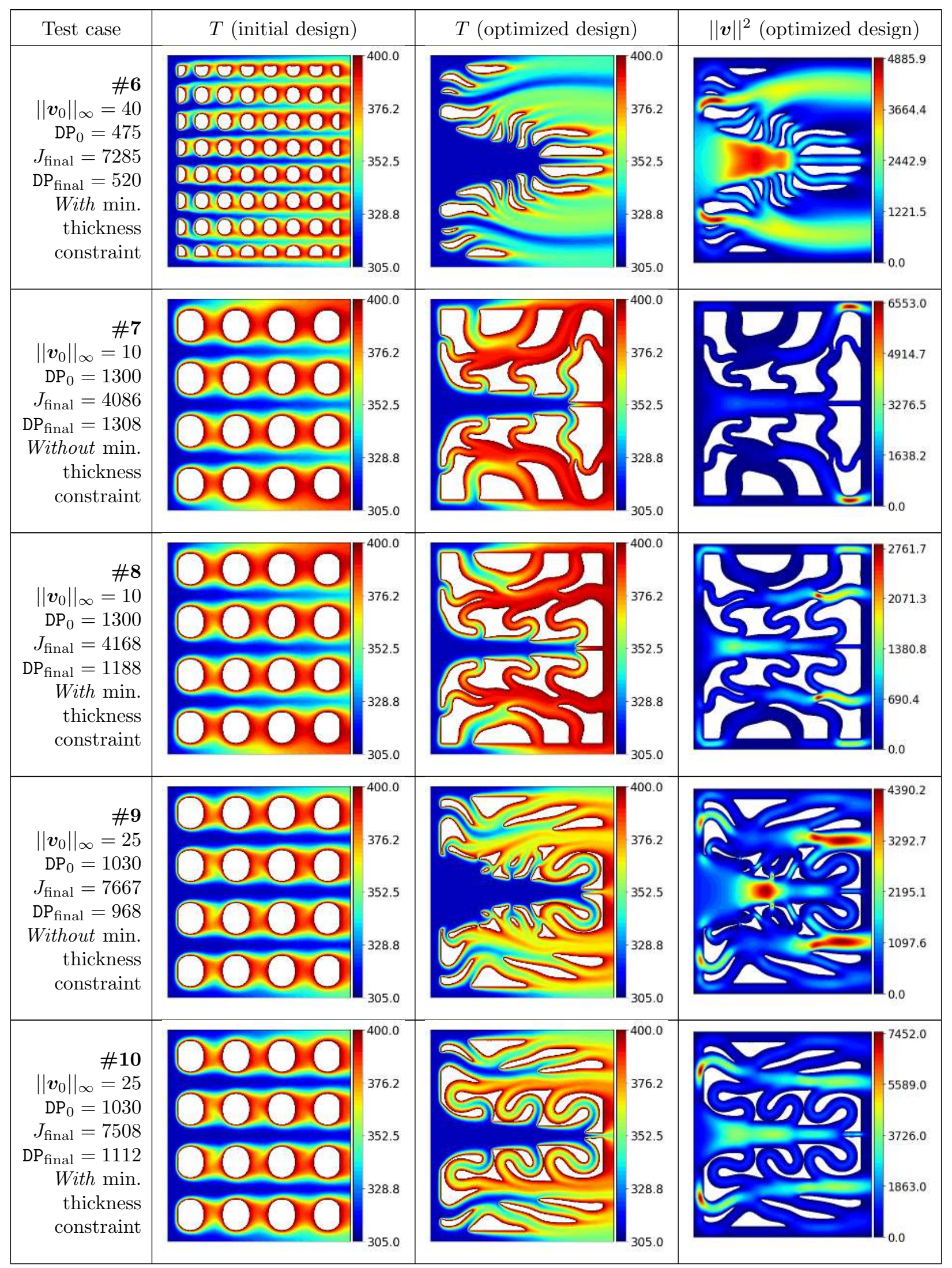




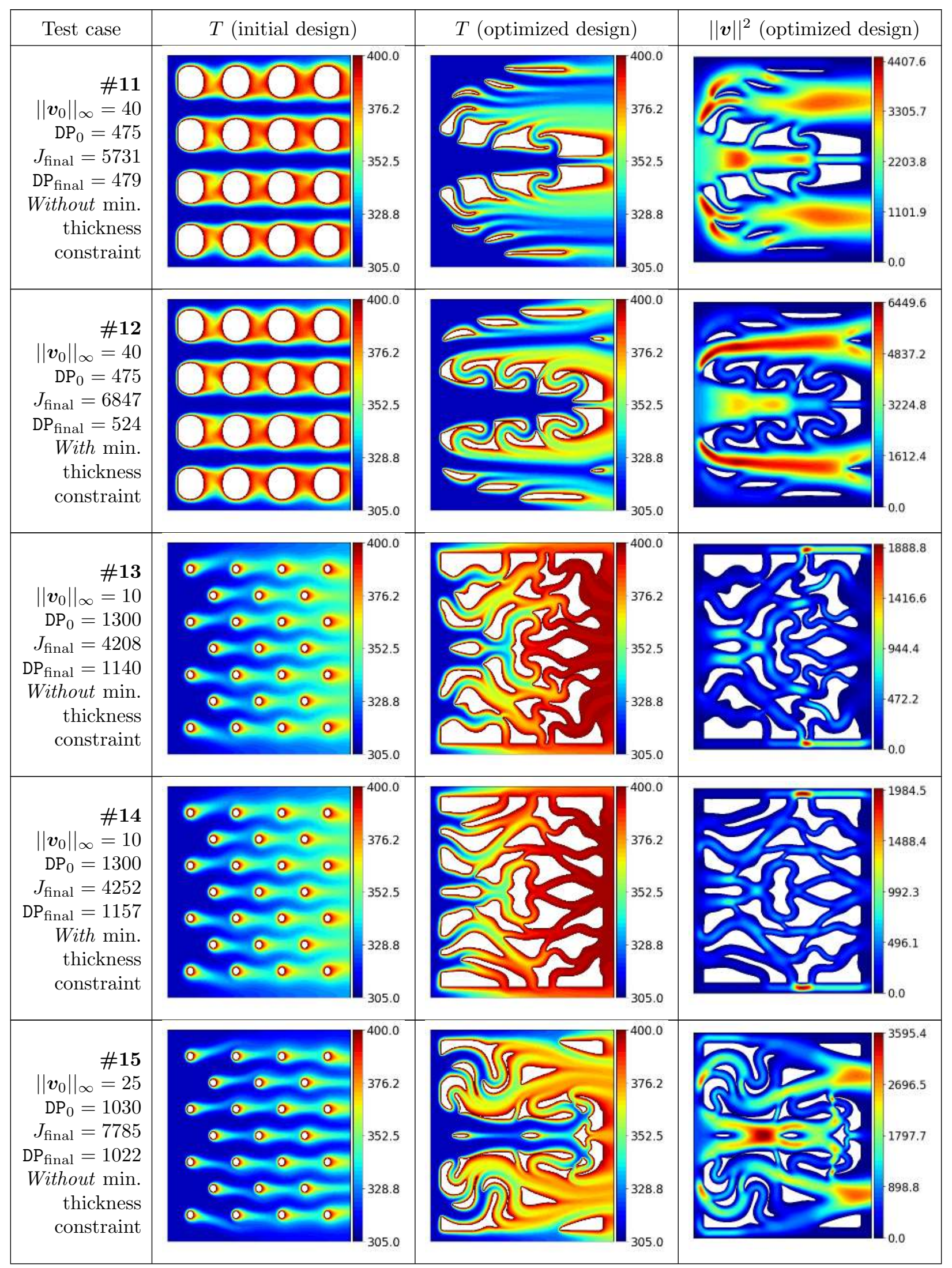




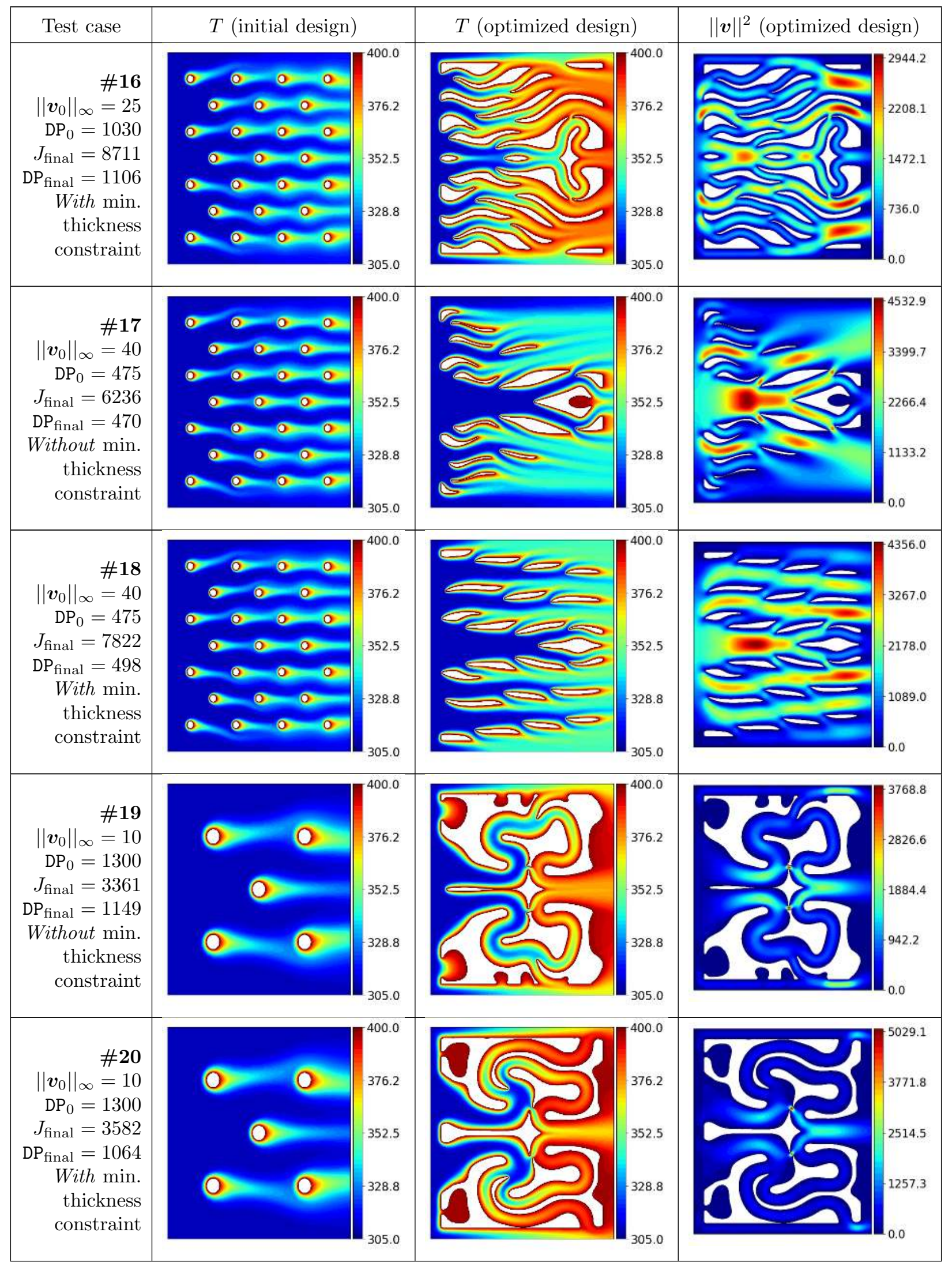




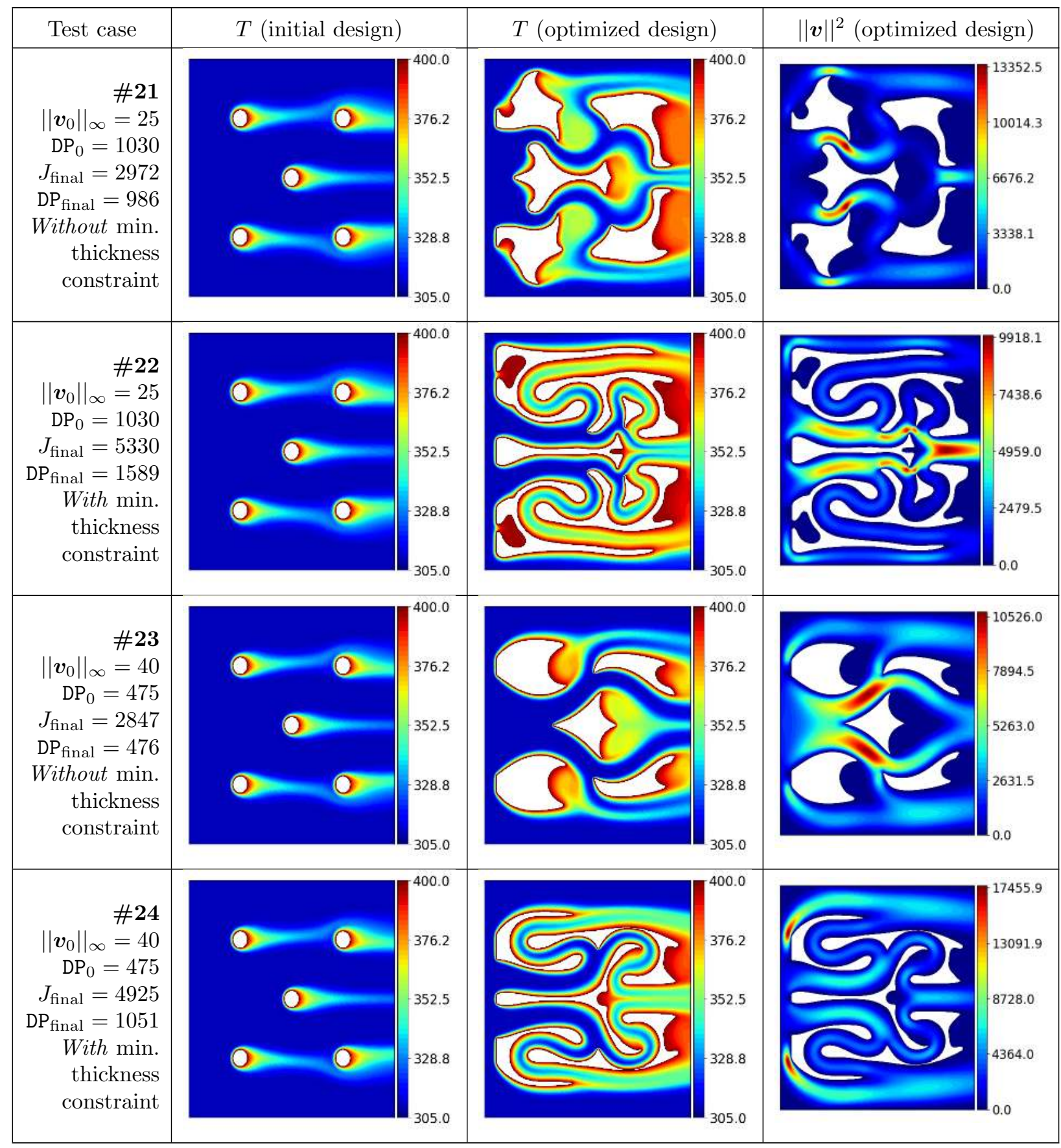

TABLE 2. Topology optimization results for the air-oil heat exchanger case study of section 3 associated to various sets of parameters and initial designs.

4. Design optimization of 2D two-tubes heat EXChangers inVolving A NON-MiXing CONSTRAint

This section and the next section 5 are devoted to the optimization of a different type of heat exchangers. These are composed of two tubes, containing two different fluids that are separated by a solid phase and must not interpenetrate. The present section is devoted to the $2 \mathrm{D}$ case, and the considered physical setting is described in section 4.1. The mathematical formulation of the optimization problem is outlined in section 4.2. 


\section{0 000 000 000}
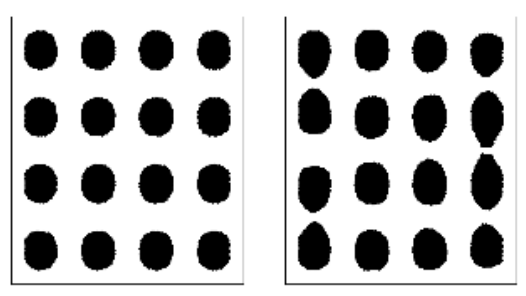

$\begin{array}{lllllllllllll}0 & 0 & 0 & 0 \\ 0 & 0 & 0 & 0 \\ 0 & 0 & 0 & 0 \\ 0 & 0 & 0 & 0 & 0\end{array}$
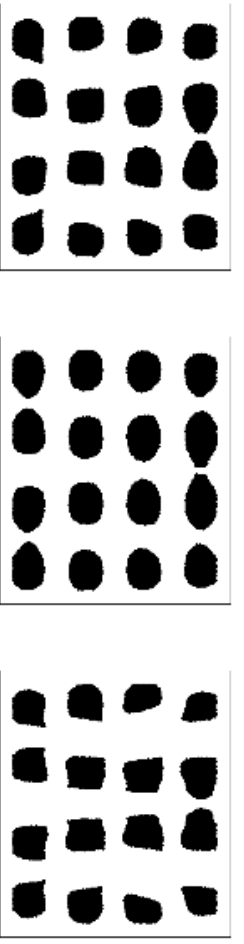
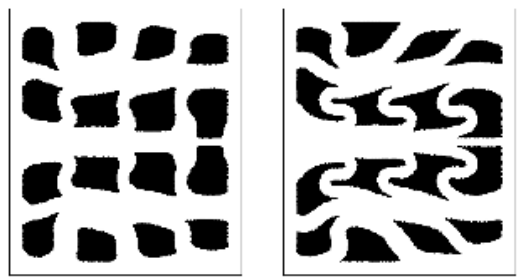

(A) Test case $8,\left\|\boldsymbol{v}_{0}\right\|_{\infty}=25$.
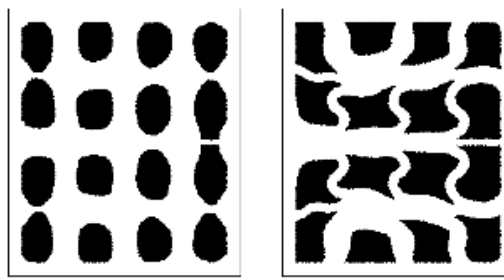

(в) Test case $10,\left\|\boldsymbol{v}_{0}\right\|_{\infty}=10$.

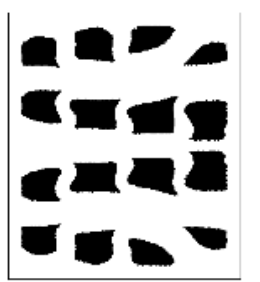

(c) Test case $12,\left\|\boldsymbol{v}_{0}\right\|_{\infty}=40$

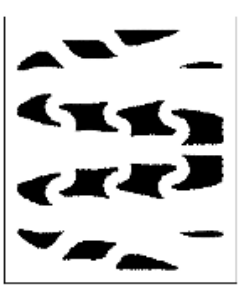

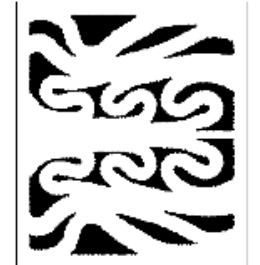
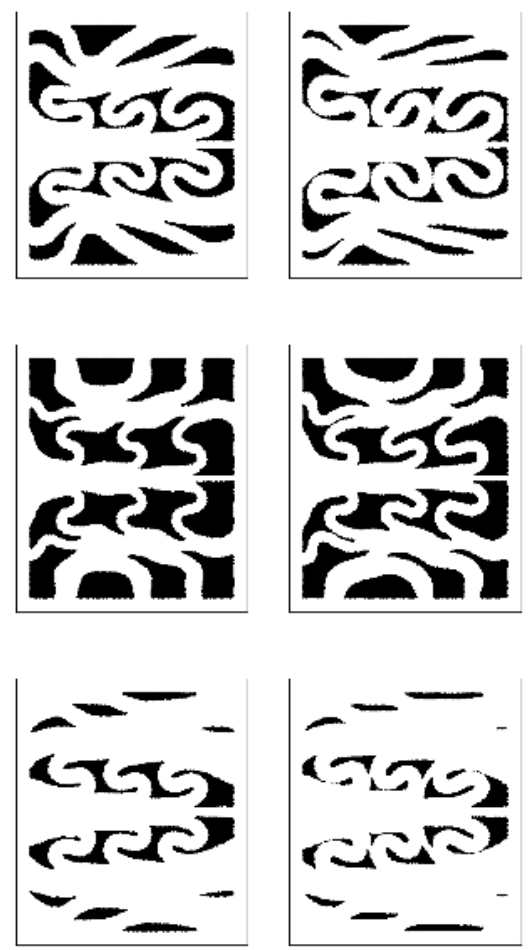

Figure 4. Iterations $0,10,20,100,200$, and 400 of the optimal design process of the 2D air-oil heat exchanger of section 3 , for several test cases.

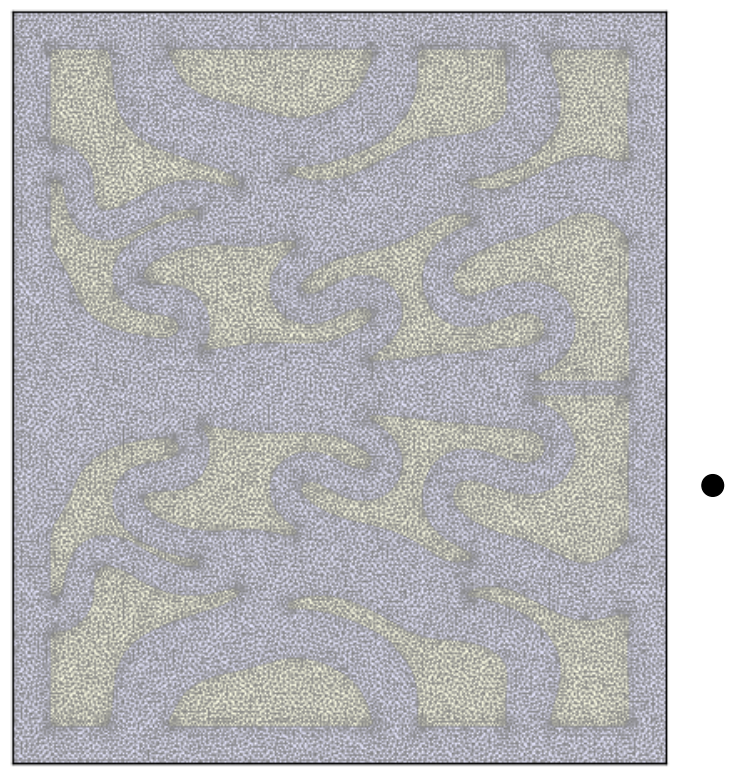

FiguRE 5. Mesh of the final shape in test case 10 of the air-oil heat exchanger optimization example of section 3. The diameter of the black circle on the right-hand side corresponds to the prescribed minimum thickness $d_{\min }=0.027$. 
10

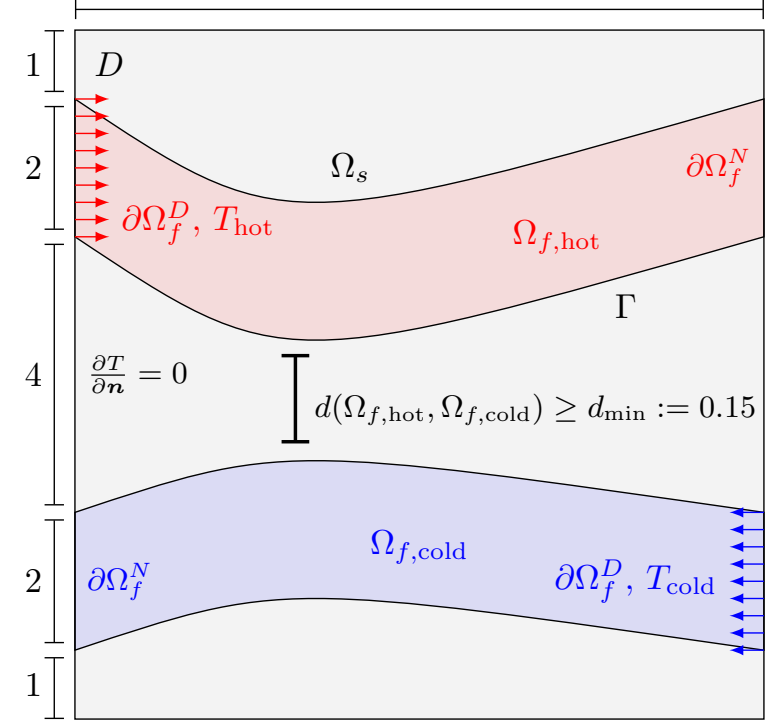

10

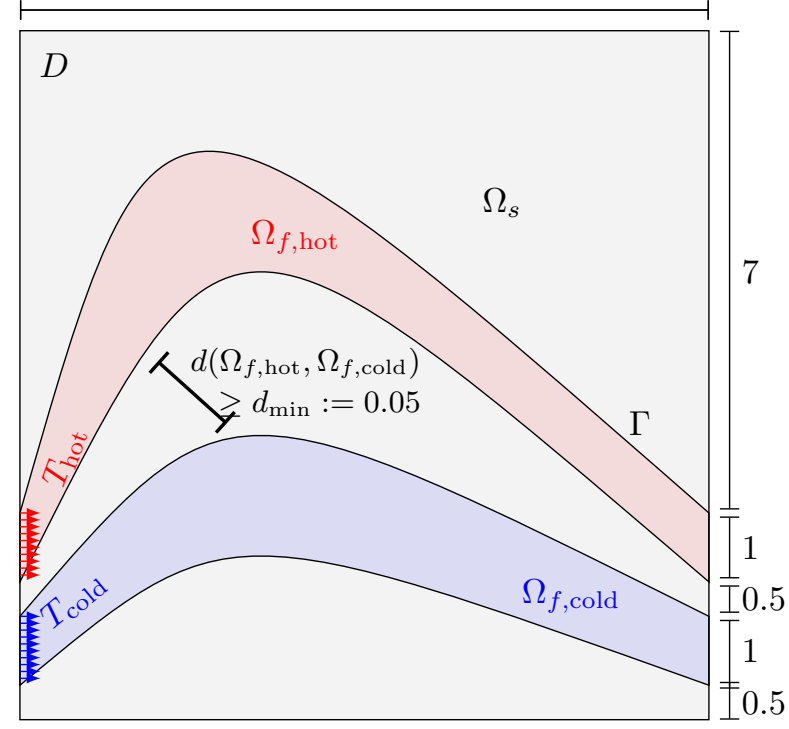

(A) Counter-current exchange test case: A hot fluid phase (в) Co-current exchange test case: a hot fluid phase $\Omega_{f \text {, hot }} \subset D$ $\Omega_{f \text {,hot }} \subset D$ is entering from the upper left side of $D$ with is entering from the upper left side of $D$ with a temperature $T_{\text {hot }}$, a temperature $T_{\text {hot }}$, and a cold fluid phase $\Omega_{f \text {,cold }}$ is entering and a cold fluid phase $\Omega_{f, \text { cold }}$ is entering in the same direction in the reverse direction from the lower right inlet. at the lower left inlet (boundary conditions not represented).

FiguRE 6. Settings of the two test cases considered in the design optimization of the heat exchangers of section 4 featuring the non-mixing condition $d\left(\Omega_{f, \text { hot }}, \Omega_{f, \text { cold }}\right) \geq d_{\min }$.

The non-penetration constraint between the fluid channels is modeled in section 4.3. Finally, numerical results are presented in section 4.4. 3D results are treated in the next dedicated section 5.

\subsection{Description of the physical setting}

In the present context, the hold-all domain is the square $D=(0,10)^{2}=\overline{\Omega_{s}} \cup \overline{\Omega_{f}}$, which is composed of the two disjoint fluid and solid phases $\Omega_{f}$ and $\Omega_{s}$, respectively. These are separated by the interface $\Gamma=\overline{\Omega_{s}} \cap \overline{\Omega_{f}}$, where the fluid satisfies the no-slip boundary condition $\boldsymbol{v}=0$. The fluid phase $\Omega_{f}=\Omega_{f \text {,hot }} \cup \Omega_{f \text {,cold }}$ itself consists of two distinct channels $\Omega_{f \text {,hot }}$ and $\Omega_{f \text {,cold }}$ whose shapes are to be optimized and kept separated. The boundary $\partial D$ of the total device $D$ contains the reunion $\partial \Omega_{f}^{D}$ of the inlets of both channels, and the reunion $\partial \Omega_{f}^{N}$ of the outlets. The hot (resp. cold) fluid is entering $D$ through $\partial \Omega_{f}^{D} \cap \partial \Omega_{f \text {,hot }}$ (resp. $\partial \Omega_{f}^{D} \cap \partial \Omega_{f, \text { cold }}$ ) with the temperature $T_{\text {hot }}=100$ (resp. $T_{\text {cold }}=0$ ) and a parabolic velocity profile $\boldsymbol{v}_{0}$. Both fluids exit $D$ with vanishing normal stress. The remaining part $\partial \Omega^{N}:=\partial D \backslash \overline{\partial \Omega_{f}^{D}}$ of the boundary of $D$ is adiabatic, bearing homogeneous Neumann condition $\frac{\partial T}{\partial \boldsymbol{n}}=0$ for the temperature field $T$. Both fluids share the same physical properties: their thermal conductivity $k_{f}$, thermal capacity $c_{p}$, kinematic viscosity $\nu$, and density $\rho$ are equal.

We consider two configurations regarding the location of the inlets, see Figure 6 for an illustration:

(i) counter-current exchange test case (Figure 6a): the two liquid phases enter $D$ from opposite sides. The inlet and outlet cross-sections share a common size $a=2$. The initial design is depicted on Figure 7 (left);

(ii) co-current exchange test case (Figure 6b): the two liquid phases enter from the same side of $D$ and the inlet and outlet cross-sections have a smaller common size $a=1$. The initial design is that on Figure 7 (right).

The physics involved in this problem are those considered in [42]: the velocity and the pressure $(\boldsymbol{v}, p)$ of the fluid obey the incompressible steady-state Navier-Stokes equations in the total fluid domain $\Omega_{f}=$ $\Omega_{f, \text { cold }} \cup \Omega_{f, \text { hot }}$ : 

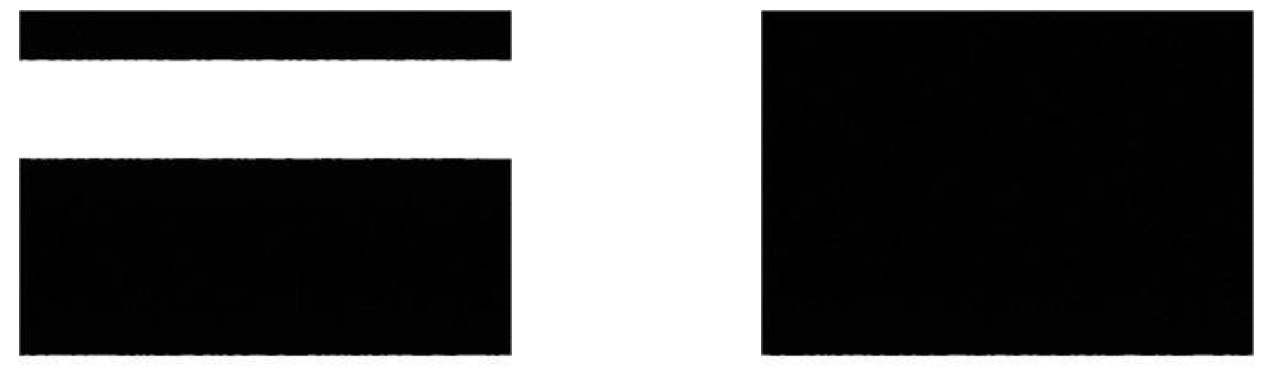

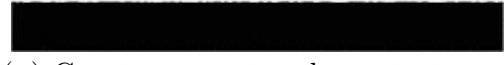

(A) Counter-current exchange test case.
(B) Co-current exchange test case.

FiguRE 7. Initial distribution of the fluid domain $\Omega_{f}^{0}$ (in white) in both test cases considered in section 4 .

$$
\left\{\begin{aligned}
-\operatorname{div}\left(\sigma_{f}(\boldsymbol{v}, p)\right)+\rho \nabla \boldsymbol{v} \boldsymbol{v} & =0 & & \text { in } \Omega_{f} \\
\operatorname{div}(\boldsymbol{v}) & =0 & & \text { in } \Omega_{f} \\
\boldsymbol{v} & =\boldsymbol{v}_{0} & & \text { on } \partial \Omega_{f}^{D} \\
\sigma_{f}(\boldsymbol{v}, p) \boldsymbol{n} & =0 & & \text { on } \partial \Omega_{f}^{N} \\
\boldsymbol{v} & =0 & & \text { on } \Gamma,
\end{aligned}\right.
$$

where the viscous stress tensor $\sigma_{f}(\boldsymbol{v}, p)$ is defined by (3.2). The temperature field $T$ is determined by the equations of convection-diffusion in the reunion of the solid and liquid phases $\Omega_{s}$ and $\Omega_{f}$ :

$$
\left\{\begin{array}{rlrl}
-\operatorname{div}\left(k_{f} \nabla T_{f}\right)+\rho c_{p} \boldsymbol{v} \cdot \nabla T_{f} & =0 & \text { in } \Omega_{f} \\
-\operatorname{div}\left(k_{s} \nabla T_{s}\right) & =0 & \text { in } \Omega_{s} \\
T & =100 & \text { on } \partial \Omega_{f}^{D} \cap \partial \Omega_{f, \text { hot }} \\
T & =0 & \text { on } \partial \Omega_{f}^{D} \cap \partial \Omega_{f, \text { cold }} \\
-k_{f} \frac{\partial T_{f}}{\partial \boldsymbol{n}} & =0 & & \text { on } \partial \Omega^{N} \cap \partial \Omega_{f} \\
-k_{s} \frac{\partial T_{s}}{\partial \boldsymbol{n}} & =0 & & \text { on } \partial \Omega^{N} \cap \partial \Omega_{s} \\
T_{f} & =T_{s} & & \text { on } \Gamma \\
-k_{f} \frac{\partial T_{f}}{\partial \boldsymbol{n}} & =-k_{s} \frac{\partial T_{s}}{\partial \boldsymbol{n}} & & \text { on } \Gamma,
\end{array}\right.
$$

where $T_{s}$ and $T_{f}$ denote the restrictions of $T$ to respectively the solid and the fluid subdomains $\Omega_{s}$ and $\Omega_{f}$.

As far as numerical values are concerned, in both test cases, the common density of the fluids is $\rho=1$, and their thermal conductivity is $k_{f}=10$. The maximum norm of the inlet velocity reads $\left\|\boldsymbol{v}_{0}\right\|_{\infty}=1$. The viscosity $\nu$ is computed by the formula $\nu:=\rho a\left\|\boldsymbol{v}_{0}\right\|_{\infty} /$ Re where the Reynolds number is set to Re $=60$. Likewise, the capacity coefficient of the fluids is calculated by $c_{p}:=k_{f} \mathrm{Pe} /(\nu \operatorname{Re})$, where the value of the Péclet number is prescribed to $\mathrm{Pe}=500$. The thermal conductivity of the solid $\Omega_{s}$ is chosen to be about ten times larger than that of the fluids: $k_{s}=110$.

\subsection{Definition of the objective functional and of the pressure drop constraint}

The aim of the optimal design problem is to find the shapes of the "hot" and "cold" components $\Omega_{f, \text { hot }}$ and $\Omega_{f \text {,cold }}$ of the fluid phase $\Omega_{f}$ that maximize the heat exchanged between both components. Two constraints are added on the design: one is about the maximum allowed pressure drop between the inlets and the outlets of the two channels, while the other imposes that $\Omega_{f \text {,cold }}$ and $\Omega_{f \text {,hot }}$ do not interpenetrate. The latter geometric constraint is discussed more thoroughly in the next section 4.3. 
The exchanged heat between both components $\Omega_{f \text {,hot }}$ and $\Omega_{f \text {,cold }}$ is mathematically appraised by the shape functional $J\left(\Omega_{f}\right) \equiv J\left(\Omega_{f}, \boldsymbol{v}\left(\Omega_{f}\right), p\left(\Omega_{f}\right), T\left(\Omega_{f}\right)\right)$ defined by

$$
J\left(\Omega_{f}\right):=-\left(\int_{\Omega_{f, \text { cold }}} \rho c_{p} \boldsymbol{v} \cdot \nabla T \mathrm{~d} x-\int_{\Omega_{f, \text { hot }}} \rho c_{p} \boldsymbol{v} \cdot \nabla T \mathrm{~d} x\right) .
$$

This quantity can indeed be interpreted as the opposite of the transferred heat since an integration by part implies that

$$
J\left(\Omega_{f}\right)=\int_{\partial \Omega_{f, \mathrm{hot}}} \rho c_{p} T \boldsymbol{v} \cdot \boldsymbol{n} \mathrm{d} s-\int_{\partial \Omega_{f, \text { cold }}} \rho c_{p} T \boldsymbol{v} \cdot \boldsymbol{n} \mathrm{d} s,
$$

where we recall that the unit normal vector $\boldsymbol{n}$ is pointing outward $D$. Since the velocity vanishes on the lateral channel boundaries, the above expression is exactly the difference between the heat conveyed by the hot phase through its output (to be minimized), and that conveyed by the cold phase (to be maximized), up to additional constant terms, depending only on the inlet boundary values (which are not subject to optimization). We favor the volume expression (4.3) which lends itself to more accurate estimates than the surface expression (4.4). This approach is rather standard in numerical fluid mechanics, see, e.g., [17, 37].

The maximum pressure drop constraint $\mathrm{DP}\left(\Omega_{f}\right)$ reads:

$$
\mathrm{DP}\left(\Omega_{f}\right):=\int_{\partial \Omega_{f}^{D}} p \mathrm{~d} s-\int_{\partial \Omega_{f}^{N}} p \mathrm{~d} s \leq \mathrm{DP}_{0},
$$

where $p \equiv p\left(\Omega_{f}\right)$ is the pressure inside the fluid and $\mathrm{DP}_{0}$ is a given threshold value. Note that there is a single constraint for the union of the two fluid components ("hot" and "cold"). In our implementation, the threshold value is set for each of the two test cases considered as follows:

$$
\mathrm{DP}_{0}:=\left\{\begin{array}{l}
2 \mathrm{DP}\left(\Omega_{f}^{0}\right) \text { in the counter-current exchange test case } \\
5 \mathrm{DP}\left(\Omega_{f}^{0}\right) \text { in the co-current exchange test case. }
\end{array}\right.
$$

Remark 3. With an abuse of notations, the objective functional (4.3), as several others in the sequel of the article, is written as a function of the fluid phase $\Omega_{f}$ while, strictly speaking, it depends on the two sub-phases $\Omega_{f, \text { hot }}$ and $\Omega_{f, \text { cold }}$.

\subsection{The non-mixing constraint between the fluid channels $\Omega_{f \text {,hot }}$ and $\Omega_{f, \text { cold }}$}

In this section, the formulation of the non-mixing condition between the two fluid phases $\Omega_{f, \text { cold }}$ and $\Omega_{f \text {,hot }}$ is discussed. Taking advantage of our numerical environment where a clear "black-and-white" and, even more, meshed representation of these phases is available at each stage of the process, this constraint is formulated in a simple geometric fashion. Our modeling is based on the signed distance function $d_{\Omega_{f, \text { hot }}}$ to $\Omega_{f \text {,hot }}$. We require that the distance between the hot and the cold phase be greater than a positive value $d_{\text {min }}$ which reads, mathematically:

or equivalently

$$
\forall x \in \Omega_{f, \text { cold }}, d_{\Omega_{f, \text { hot }}}(x) \geq d_{\text {min }},
$$

$$
\forall x \in \partial \Omega_{f, \text { cold }}, \quad d_{\Omega_{f, \text { hot }}}(x) \geq d_{\min } .
$$

Obviously, the roles of $\Omega_{f \text {,hot }}$ and $\Omega_{f \text {,cold }}$ can be interchanged in (4.5) and (4.6). The minimum distance parameter is set to $d_{\min }=0.15$ for the counter-current exchange test case and $d_{\min }=0.05$ for the cocurrent one. Following the strategy in $[10,68]$, we then construct averaged-penalty functionals in order to approximate the pointwise constraint (4.6). To this end, we first note that (4.6) is equivalent to the following requirement:

$$
\left\|\frac{1}{d_{\Omega_{f, \text { hot }}}}\right\|_{L^{\infty}\left(\partial \Omega_{f, \text { cold })}\right.}^{-1} \geq d_{\min }
$$

We then classically approximate the supremum norm in the above equation with an $L^{p}$ norm, for a sufficiently large value of $p$, so as to obtain a differentiable shape functional. Our approximate version of (4.6) reads:

$$
P_{\text {cold } \rightarrow \text { hot }}\left(\Omega_{f}\right) \geq d_{\text {min }}, \text { where } P_{\text {cold } \rightarrow \text { hot }}\left(\Omega_{f}\right):=\left\|\frac{1}{d_{\Omega_{f, \text { hot }}}}\right\|_{L^{p}\left(\partial \Omega_{f, \text { cold }}\right)}^{-1}=\left(\int_{\partial \Omega_{f, \text { cold }}} \frac{1}{\left|d_{\Omega_{f, \text { hot }}}\right|^{p}} \mathrm{~d} s\right)^{-\frac{1}{p}} \text {. }
$$


The quantity $P_{\text {cold } \rightarrow \text { hot }}\left(\Omega_{f}\right)$ can be interpreted as an averaged measure of the distance $d\left(\Omega_{f, \text { cold }}, \Omega_{f, \text { hot }}\right)$ between both phases. In our implementation, the parameter $p$ involved is set to $p=4$.

Remark 4. A similar average criterion to (4.8) could have been constructed from (4.5) as an integral over the whole cold phase $\Omega_{f, \text { cold }}$, and not only the boundary $\partial \Omega_{f \text {,cold }}$. We nevertheless prefer the formulation (4.6) and (4.8) because we expect that averaging on a smaller set in (4.8) yields a more accurate approximation of the infinity norm.

Remark 5. The constraint (4.7) on the distance between the two connected components $\Omega_{f, \text { cold }}$ and $\Omega_{f \text {,hot }}$ amounts to imposing that there be a solid wall of minimum thickness $d_{\min }$ between the two phases. It is quite different (and easier) from the requirement of a minimum thickness for the whole solid phase $\Omega_{s}$ (which we do not aim to impose).

In order to balance the effect of the constraint (4.8) over both fluid phases, we introduce the symmetric counterpart of (4.8):

$$
P_{\text {hot } \rightarrow \text { cold }}\left(\Omega_{f}\right):=\left(\int_{\partial \Omega_{f, \text { hot }}} \frac{1}{\mid d_{\Omega_{f, \text { cold }} \mid p}} \mathrm{~d} s\right)^{-\frac{1}{p}} .
$$

Eventually, we consider the symmetrized non-penetration constraint obtained by the harmonic mean of $P_{\text {hot } \rightarrow \text { cold }}$ and $P_{\text {cold } \rightarrow \text { hot }}$. Our non-mixing constraint (4.7) finally reads:

$$
Q_{\text {hot } \leftrightarrow \text { cold }}\left(\Omega_{f}\right) \geq d_{\text {min }}, \text { where } Q_{\text {hot } \leftrightarrow \text { cold }}\left(\Omega_{f}\right):=\frac{2}{1 / P_{\text {hot } \rightarrow \text { cold }}\left(\Omega_{f}\right)+1 / P_{\text {cold } \rightarrow \text { hot }}\left(\Omega_{f}\right)} .
$$

All in all, the considered optimization problem in this $2 \mathrm{D}$ setting is:

$$
\begin{gathered}
\min _{\Omega_{f} \subset D} J\left(\Omega_{f}\right)=-\left(\int_{\Omega_{f, \text { cold }}} \rho c_{p} \boldsymbol{v} \cdot \nabla T \mathrm{~d} x-\int_{\Omega_{f, \text { hot }}} \rho c_{p} \boldsymbol{v} \cdot \nabla T \mathrm{~d} x\right) \\
\text { s.t. }\left\{\begin{array}{c}
\mathrm{DP}\left(\Omega_{f}\right)=\int_{\partial \Omega_{f}^{D}} p \mathrm{~d} s-\int_{\partial \Omega_{f}^{N}} p \mathrm{~d} s \leq \mathrm{DP}_{0} \\
Q_{\text {hot } \leftrightarrow \text { cold }}\left(\Omega_{f}\right) \geq d_{\text {min }} .
\end{array}\right.
\end{gathered}
$$

Again, the shape derivatives of the "physical" functionals $J\left(\Omega_{f}\right)$ and $\operatorname{DP}\left(\Omega_{f}\right)$ are computed thanks to the formulas provided in our previous work [42], while that of the geometric constraint functional $Q_{\text {hot } \leftrightarrow \text { cold }}\left(\Omega_{f}\right)$ is evaluated numerically thanks to the variational method presented in section 2.4.

\subsection{Numerical results}

The optimization problem (4.11) is solved with the numerical strategy described in section 2 and summarized in algorithm 1. The obtained results for both test cases 1 and 2 of Figure 6 are depicted on Figs. 8 to 11 and Figs. 13 to 16, respectively. In each situation, we display the initial and final designs, the temperature $T$ and the velocity field $\boldsymbol{v}$ within the latter, as well as several intermediate shapes obtained with our algorithm and the convergence histories for the objective and constraint functionals. Both constraints (minimum distance and maximum pressure drop) are saturated at convergence. The resulting serpentine shapes are similar to those observed in [76].

Remark 6. The convergence histories of Figs. 11 and 16 reveal that the design obtained at convergence is not the "best" one among all those obtained during the optimization process, in terms of the objective function $J\left(\Omega_{f}\right)$. Indeed, the intermediate designs at iteration 100 and 150 are slightly better than the final ones, in the context of the counter-current and the co-current heat exchanger test case, respectively. This fact can be explained with the treatment of the kind of solid "crack" (or thin wall) featured by these intermediate designs, by means of the remeshing process of our optimization methodology. The latter indeed attempts to find a compromise between the physical performance of the shape, of its smoothness, and the enforcement of minimum and maximum mesh size constraints. As a consequence, the "cracks" appearing in the corners of the fluid pipes are smoothed or even broken in the course of the remeshing process (see for instance the iteration 155 of Figure 15 for the co-current heat exchanger test case), which results in a slight degradation of the physical performance of the shapes. 


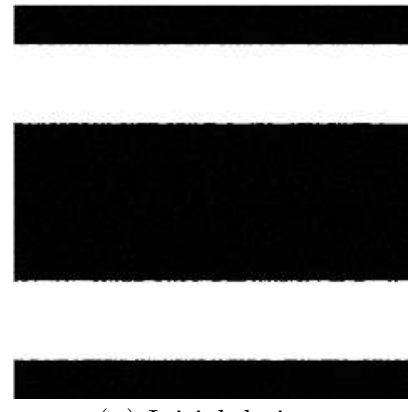

(A) Initial design.

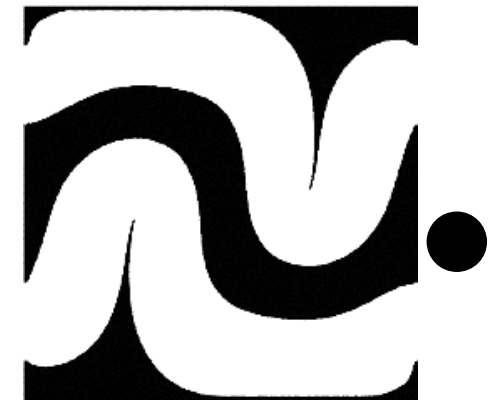

(в) Optimized design.

FiguRE 8. Initial and final designs for the counter-current exchange test case of section 4. The diameter of the black disk on the right-hand side indicates the value of the minimum distance constraint.

Remark 7. The discrepancy between the pressure drop ratios $\operatorname{DP}\left(\Omega_{f}\right) / \operatorname{DP}\left(\Omega_{f}^{0}\right)$ attained at convergence (1.7 and 4.7 in respectively Figs. 11 and 16) and the imposed upper bounds (which equal 2 and 5 for the counter-current and the co-current test cases, respectively) can be accounted for by the high sensitivity of the pressure constraint to rather small variations of the domain in the vicinity of the tip of its solid "crack" (or thin wall). Indeed, Remark 8 in [43] suggests an a posteriori tolerance bound for the fulfillment of this constraint. Because of the imposed minimum mesh size $h_{\min }$, one can only expect that, at convergence, the optimized domain $\Omega_{f}$ satisfies the constraint up to a deformation field $\boldsymbol{\theta} \in W^{1, \infty}\left(D, \mathbb{R}^{d}\right)$ with magnitude $\|\boldsymbol{\theta}\|_{L^{\infty}(D)} \approx h_{\min }$. Therefore, the uncertainty over the values of the constraint functional $C\left(\Omega_{f}\right)$ is of the order:

$$
\max _{\|\boldsymbol{\theta}\|_{L}(D) \leq h_{\min }}\left|\mathrm{D} C\left(\Omega_{f}\right) \cdot(\boldsymbol{\theta} \cdot \boldsymbol{n})\right|=\max _{\|\boldsymbol{\theta}\|_{L} \infty(D) \leq h_{\min }}\left|\int_{\partial \Omega_{f}} v_{C}\left(\Omega_{f}\right) \boldsymbol{\theta} \cdot \boldsymbol{n} \mathrm{d} s\right| \leq \varepsilon:=h_{\min } \int_{\partial \Omega_{f}}\left|v_{C}\left(\Omega_{f}\right)\right| \mathrm{d} s,
$$

where $v_{C}\left(\Omega_{f}\right)$ is the scalar field featured in the surface expression (2.6) of the shape derivative of the pressure drop constraint. At convergence, we find numerically $\varepsilon \simeq 5$ and $\varepsilon \simeq 60$ for respectively the co-current and counter-current heat exchanger test cases. Therefore, the achieved discrepancy of order 0.3 seems acceptable with respect to these bounds.

The optimized shapes suggest two comments. At first, our approximation (4.8) of the infinity norm by the $L^{p}$ norm with $p=4$ works well: the distance constraint is clearly respected at almost all intermediate iterations. Secondly, our method is able to improve continuously the optimized design even after the saturation of the distant constraint, which happens very early in the optimization process.

Finally, let us mention that our computations do not require a very fine mesh of the solid region $\Omega_{s}$ lying between the cold and the hot domains $\Omega_{f, \text { cold }}$ and $\Omega_{f, \text { hot }}$ so as to properly handle the distance constraint (4.10). As an illustration of this fact, we plot on Figs. 12 and 17 the final meshes of the optimized shape for both test cases. For the co-current exchange test case, a resolution of about ten mesh elements (that is, the skeleton $\Sigma$ of $\Omega_{f}$ lies at approximately five mesh elements from the boundary $\partial \Omega_{f}$ only) in between the two pipes proves sufficient to obtain a satisfactory approximation of the shape derivative of the geometric constraint functional $Q_{\text {hot↔cold }}\left(\Omega_{f}\right)$ from the variational method of section 2.4.

\section{Shape And topology optimization of a 3D Fluid-TO-Fluid HeAt EXCHANGER INVOLVING A NON-MIXING CONSTRAINT}

In this section, the optimal design of a three-dimensional two-tube heat exchanger is investigated. The physical setting is the three-dimensional counterpart of that described in section 4.1. Here, the hold-all domain is the cube $D=(0,1)^{3}$, as in Figure 18. A cold channel is flowing in the $x$-direction from a diskshaped inlet of radius $a=0.1$ located on the left face of $\partial D$, and is exiting through a disk with the same radius $a$ located on the opposite face. A hot channel is flowing in the $z$-direction between similar disk-shaped 


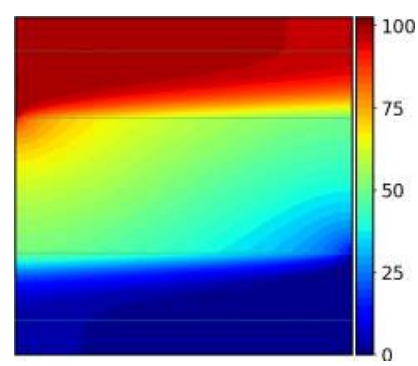

(A) Initial temperature field.

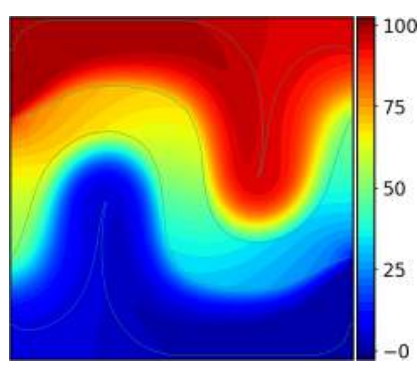

(в) Final temperature field.

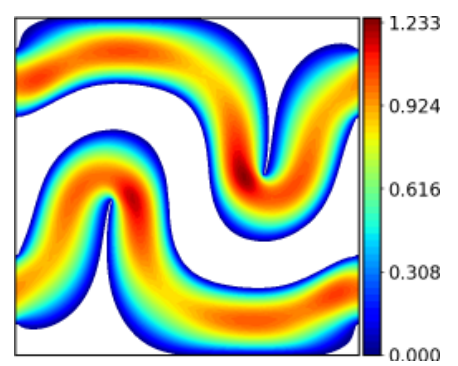

(c) Final norm of the fluid velocity.

Figure 9. Plots of the state variables at the last iteration for the co-current exchange test case of section 4 .

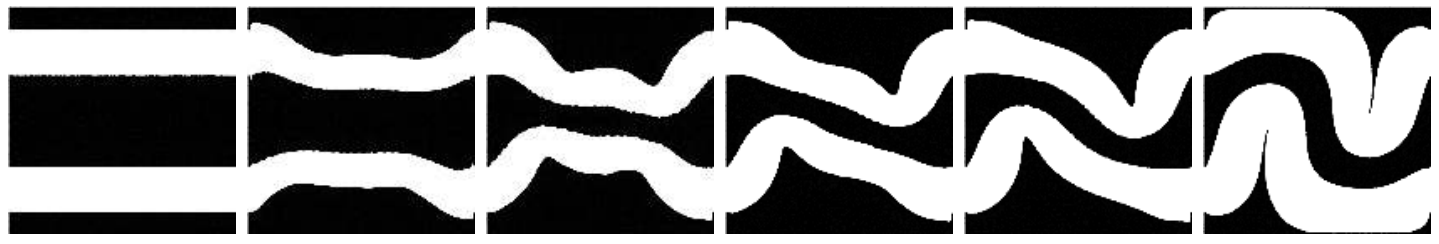

Figure 10. Intermediate iterations $0,10,20,30,40$, and 400 for the counter-current exchange test case of section 4 .

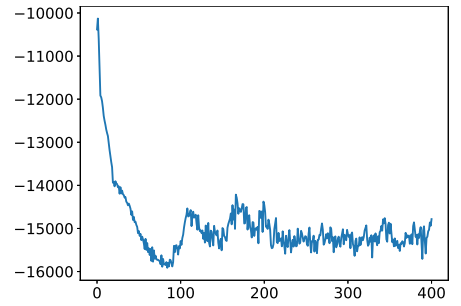

(A) Objective function $J\left(\Omega_{f}\right)$. Final value: $J\left(\Omega_{f}\right)=-15,000$.

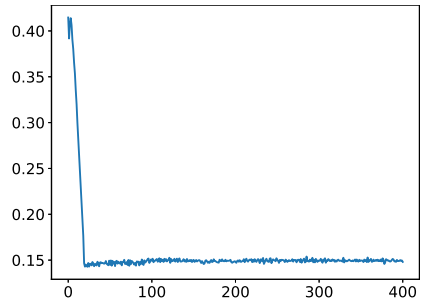

(в) Penalty functional $Q_{\text {cold } \leftrightarrow \text { hot }}$ for the non-mixing constraint. Final value: $Q_{\text {cold } \leftrightarrow \text { hot }}\left(\Omega_{f}\right)=0.15$.

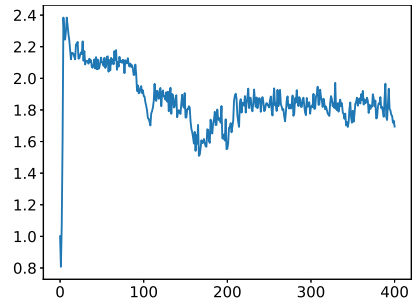

(C) Pressure drop $\mathrm{DP}\left(\Omega_{f}\right) / \mathrm{DP}\left(\Omega_{f}^{0}\right)$. Final value: $\operatorname{DP}\left(\Omega_{f}\right) / \operatorname{DP}\left(\Omega_{f}^{0}\right)=1.7$.

FigURE 11. Convergence histories for the counter-current exchange test case of section 4.
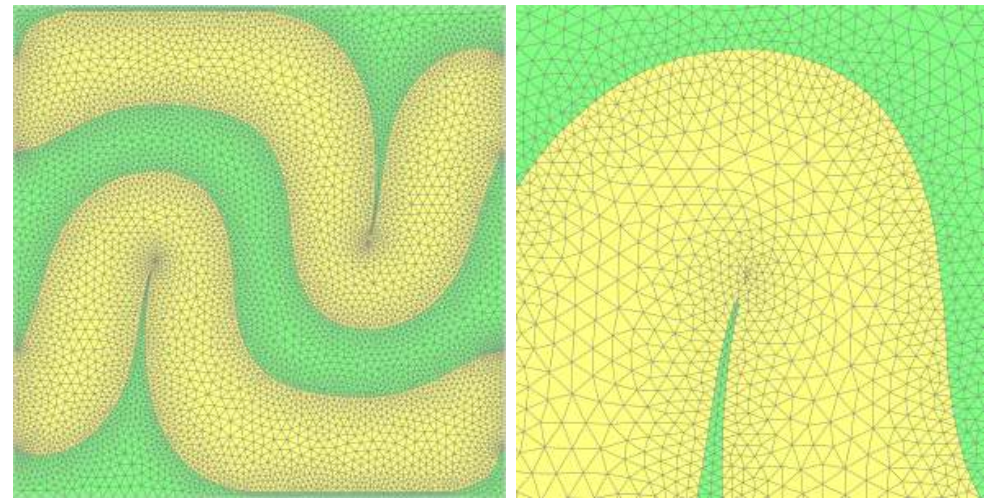

FigURE 12. Zoom on a mesh of an intermediate optimization iteration occurring during the solution of the counter-current exchange test case of section 4 . 


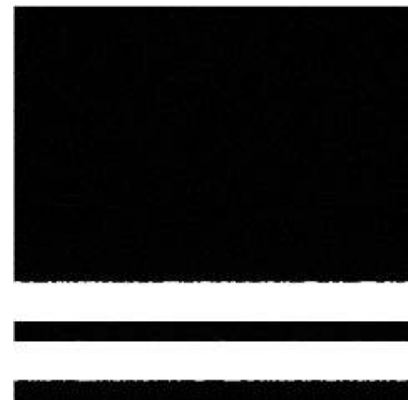

(A) Initial design.

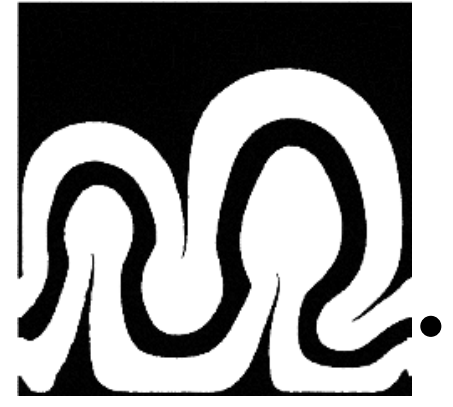

(в) Optimized design.

FiguRE 13. Initial and final designs for the co-current exchange test case of section 4. The diameter of the black circle on the right-hand side indicates the value of the minimum distance constraint.

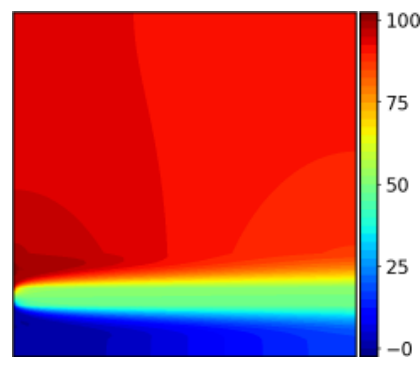

(A) Initial temperature field.

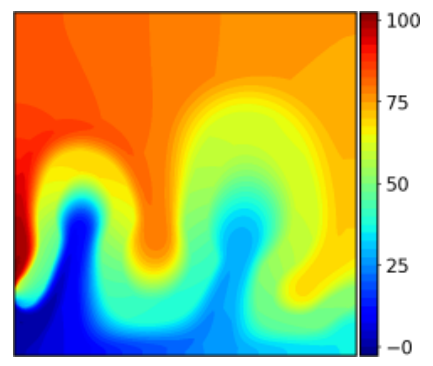

(в) Final temperature field.

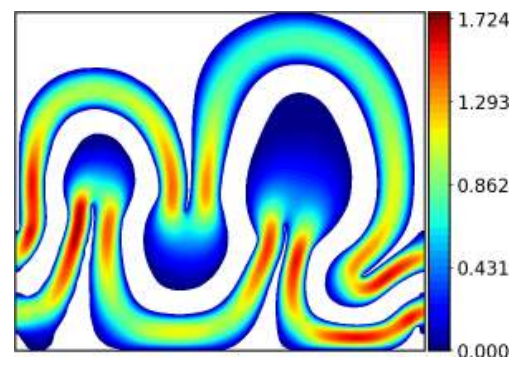

(c) Final norm of the fluid velocity.

Figure 14. Plots of the state variables at the last iteration for the co-current exchange test case of section 4 .
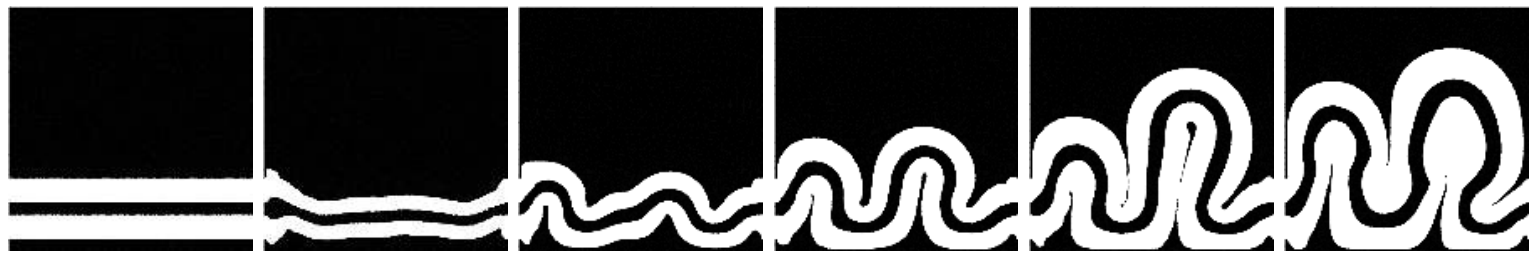

FiguRE 15. Intermediate iterations $0,15,50,88,155$ and 400 in the co-current exchange test case of section 4.

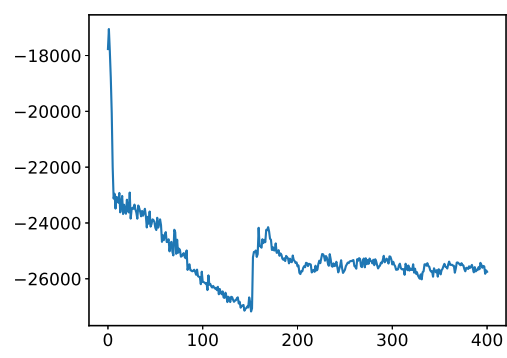

(A) Objective function $J\left(\Omega_{f}\right)$. Final value: $J\left(\Omega_{f}\right)=-26,000$.

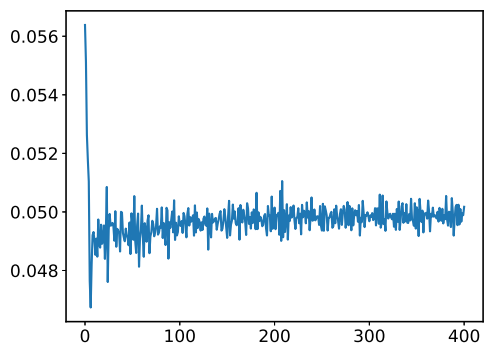

(B) Penalty functional $Q_{\text {cold } \leftrightarrow \text { hot }}$ for the non-mixing constraint. Final value: $Q_{\text {cold } \leftrightarrow \text { hot }}\left(\Omega_{f}\right)=0.05$.

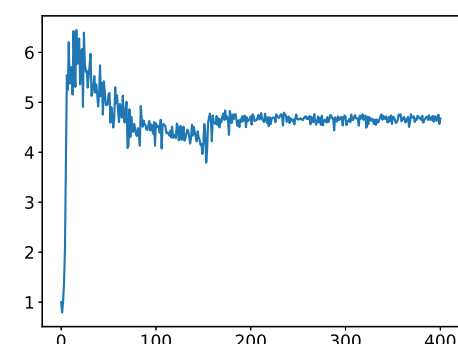

(C) Pressure drop $\operatorname{DP}\left(\Omega_{f}\right) / \mathrm{DP}\left(\Omega_{f}^{0}\right)$. Final value: $\operatorname{DP}\left(\Omega_{f}\right) / \operatorname{DP}\left(\Omega_{f}^{0}\right)=4.7$.

Figure 16. Convergence histories for the co-current exchange test case (where the cold and hot inlets are located on the same side of $\partial D$ ) of section 4. 

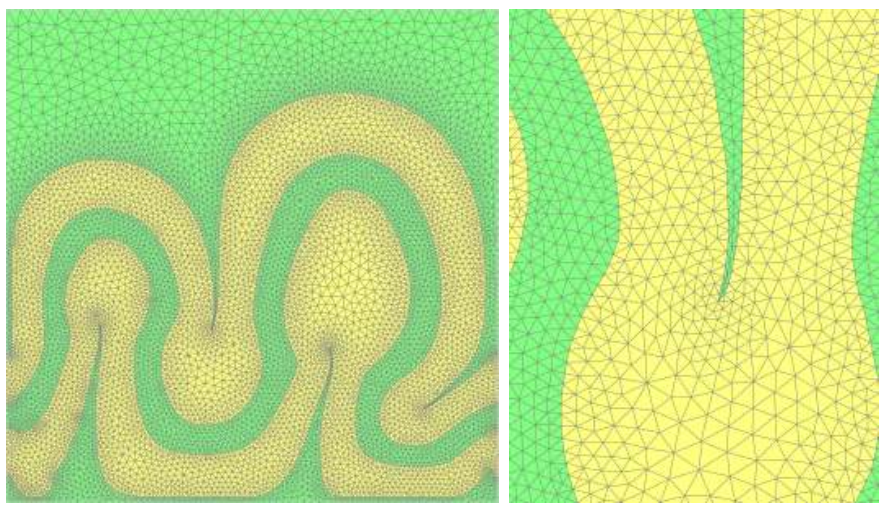

FIGURE 17. Zoom on a mesh of an intermediate optimization iteration occurring during the solution of the co-current exchange test case of section 4 .

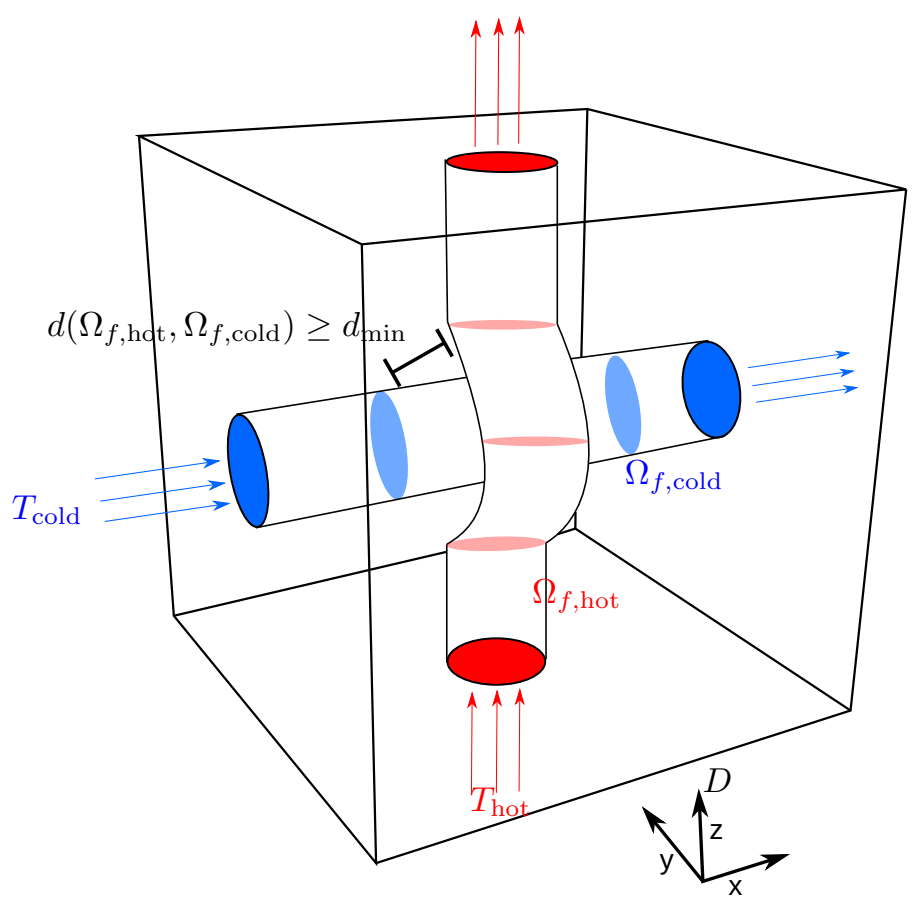

FIGURE 18. Schematic of the 3D setting of section 5 .

input and output located on the bottom and top faces of $\partial D$. The input temperatures for the hot and cold channels are the same as in the previous $2 \mathrm{D}$ case: $T_{\text {hot }}=100\left(\right.$ resp. $\left.T_{\text {cold }}=0\right)$.

Similarly to the 2D case, we solve the Navier-Stokes system (4.1) and the convection-diffusion equation (4.2) for the velocity, pressure, and temperature fields $(\boldsymbol{v}, p, T)$. This step is achieved in FreeFEM [50] and the parallel preconditioning features of PETSc [16, 15], see [45] where the implementation is described in details. The values of the physical parameters featured in there are reported in Table 3. After describing the considered shape optimization problem in the present 3D situation in section 5.1, we discuss in sections 5.2 and 5.3 a few numerical issues that are quite specific to the present three-dimensional context, before turning to numerical results in section 5.4. 


\begin{tabular}{ccccccccc}
\hline $\mathrm{Re}$ & $d_{\min }$ & $\rho$ & $k_{f}$ & $k_{s}$ & $\mathrm{Pe}$ & $c_{p}$ & $\mathrm{DP}_{0}$ & $\mathrm{~V}_{0}$ \\
\hline 100 & 0.1 or 0.04 & 10 & 1 & 10 & 2,000 & 200 & 3.72 & 0.15 \\
\hline
\end{tabular}

TABLE 3. Numerical values of the physical parameters for the $3 \mathrm{D}$ setting of section 5 .

\subsection{Formulation of the optimization problem}

Although very similar in spirit, the three-dimensional character of the shape and topology optimization problem addressed in this section raises the need for several adjustments in the formulation of the 2D problem treated in section 4.

At first, topological changes are much more likely to occur in 3D than in 2D. Thus, it becomes crucial for our purpose to enforce strictly a maximum pressure loss constraint on each of the two channels in order to prevent them from becoming disconnected. Indeed, the thermal conductivity $k_{s}$ in the solid phase $\Omega_{s}$ is larger than $k_{f}$ in the fluid phase, and so the algorithm may accidentally find it beneficial to reduce too much the pipe cross-sections to favor the transport of heat through diffusion. Therefore, for an increased robustness of the optimization path selected by our null space algorithm, we consider two static pressure loss constraints in the minimization problem (5.1) below, one for each of the channels $\Omega_{f \text {,hot }}$ and $\Omega_{f \text {,cold }}$, instead of just one in the whole fluid phase $\Omega_{f}$ as in the $2 \mathrm{D}$ case.

In the same spirit, we express our minimum distance constraint between the two channels as two constraints $P_{\text {hot } \rightarrow \text { cold }}\left(\Omega_{f}\right)$ and $P_{\text {cold } \rightarrow \text { hot }}\left(\Omega_{f}\right)$ given in (4.8) and (4.9) instead of considering the symmetrized quantity $Q_{\text {hot } \leftrightarrow \text { cold }}$ from (4.10). Although slightly redundant, this formulation tends to impose the distance constraint in a more stringent way, while still attributing symmetric roles to $\Omega_{f \text {,hot }}$ and $\Omega_{f, \text { cold }}$. Finally, we add to the problem a constraint on the volume of each of the two channels in order to ensure that these are balanced in mass.

All things considered, the optimal design problem of interest in this section reads:

$$
\begin{aligned}
& \min _{\Omega_{f} \subset D} J\left(\Omega_{f}\right)=-\left(\int_{\Omega_{f, \text { cold }}} \rho c_{p} \boldsymbol{v} \cdot \nabla T \mathrm{~d} x-\int_{\Omega_{f, \text { hot }}} \rho c_{p} \boldsymbol{v} \cdot \nabla T \mathrm{~d} x\right) \\
& \text { s.t. }\left\{\begin{aligned}
& \operatorname{Vol}\left(\Omega_{f, \text { hot }}\right):=\int_{\Omega_{f, \text { hot }}} \mathrm{d} x \leq \mathrm{V}_{0} \\
& \operatorname{Vol}\left(\Omega_{f, \text { cold }}\right):=\int_{\Omega_{f, \text { cold }}} \mathrm{d} x \leq \mathrm{V}_{0} \\
& \operatorname{DP}\left(\Omega_{f, \text { hot }}\right)= \int_{\partial \Omega_{f, \text { hot }}^{D}} p \mathrm{~d} s-\int_{\partial \Omega_{f, \text { hot }}^{N}} p \mathrm{~d} s \leq \mathrm{DP}_{0} \\
& \operatorname{DP}\left(\Omega_{f, \text { cold }}\right)=\int_{\partial \Omega_{f, \text { cold }}^{D}} p \mathrm{~d} s-\int_{\partial \Omega_{f, \text { cold }}^{N}} p \mathrm{~d} s \leq \mathrm{DP}_{0} \\
& P_{\text {hot } \rightarrow \text { cold }}\left(\Omega_{f}\right) \geq d_{\text {min }} \\
& P_{\text {cold } \rightarrow \text { hot }}\left(\Omega_{f}\right) \geq d_{\text {min }},
\end{aligned}\right.
\end{aligned}
$$

where we have defined $\partial \Omega_{f, \text { hot }}^{D}:=\partial \Omega_{f}^{D} \cap \partial \Omega_{f, \text { hot }}, \partial \Omega_{f, \text { cold }}^{D}:=\partial \Omega_{f}^{D} \cap \partial \Omega_{f, \text { cold }}$, and likewise for $\partial \Omega_{f, \text { hot }}^{N}$ and $\partial \Omega_{f, \text { cold }}^{N}$.

\subsection{Selection of an initial design}

One crucial point in the solution of shape and topology optimization problems in the framework of Hadamard's method is the choice of an adequate initial design. In the present physical context, the initial shapes of $\Omega_{s}$, $\Omega_{f \text {,hot }}$, and $\Omega_{f \text {,cold }}$ have to comply with the topological constraints of two-fluid heat exchangers, namely:

- the cold and hot inputs of the fluid subdomain $\Omega_{f}$ must be connected to their associated outputs;

- the initial fluid subdomain has to be the disjoint reunion $\Omega_{f}=\Omega_{f \text {,cold }} \cup \Omega_{f \text {,hot }}$ of the hot and cold phases. 

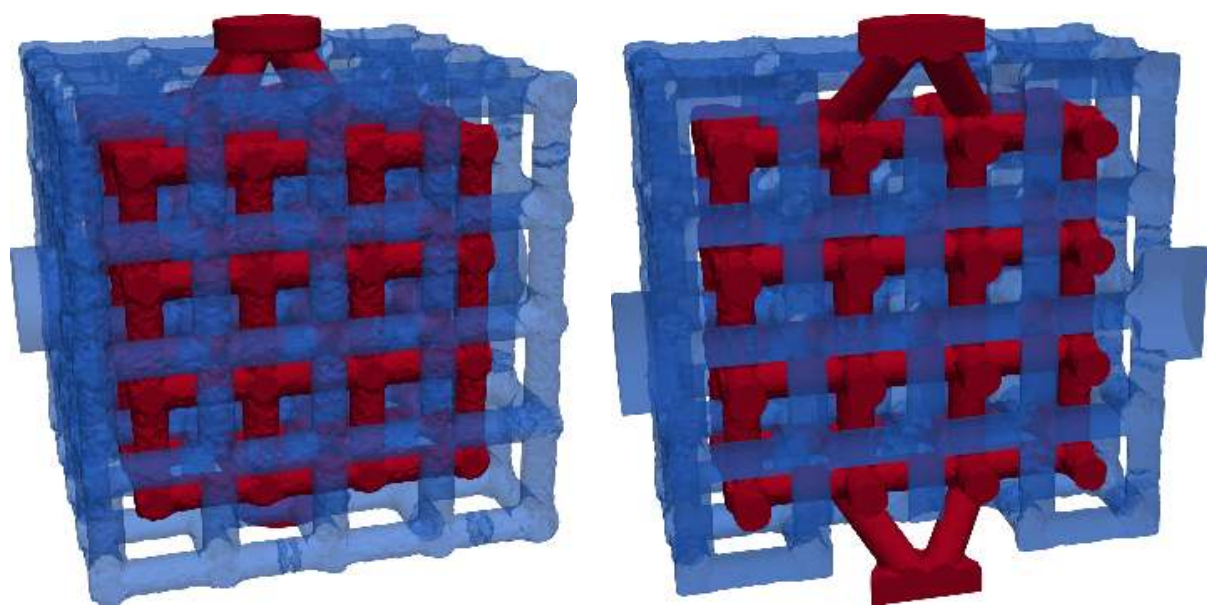

FigURE 19. Initial distribution of fluid considered for the 3D heat exchanger test case of section 5. The hot and cold phase are depicted in red and blue respectively and are disjoint regions. Cut with respect to the $z$-axis on the right.

In two space dimensions, these requirements do not leave room for many changes in the topology with respect to the initial setting, which naturally resembles those depicted on Figure 7 . The only possible topological enrichment would be the addition of solid inclusions in each of the fluid phases. In the present three-dimensional situation, there is much more freedom about the selection of the initial shapes of $\Omega_{s}$, $\Omega_{f \text {,hot }}$, and $\Omega_{f \text {,cold }}$. In the implementation, we consider two nested but non-mixing cell arrays of pipes which are connected to their respective inputs and outputs. This initial design is displayed on Figure 19. Its construction is achieved by means of simple $\mathrm{min} / \max$ operations on primitive level-set functions associated to elementary patterns (e.g., cylinders, cubes, or spheres), corresponding respectively to union/intersection of their associated subdomain [72]. The initial design is chosen with a rich enough topology of channels because the minimum distance constraint will not allow for the crossing of cold and hot channels.

Note that although both hot and cold phases should constitute two distinct connected components at the initialization, the condition that both phases be separated from a minimum distance $d_{\min }$ does not need to be strictly satisfied: these violations of the optimization constraints are automatically corrected by the null space algorithm from section 2.3 at subsequent iterations.

\subsection{Numerical solution of the topology optimization problem}

The overall methodology for the solution of the heat exchanger design problem (5.1) is identical to that of the 2D case of section 4 and it relies on all theoretical ingredients recalled in section 2. However, the threedimensional character of the problem requires specific implementation changes regarding mesh operations and the solution of linear systems.

Remeshing operations and isosurface discretization on tetrahedral meshes are notoriously more computationally expensive in $3 \mathrm{D}$ than in 2D. These are performed thanks to the open-source library Mmg [27, 26] in our implementation. For now, this task is sequential, however it should be possible to perform it in parallel in the near future with the next version of ParMmg [24].

Then, the large dimension of the finite element systems at stake is overcome by parallel computing with the help of modern domain decomposition techniques and suitable preconditioning. For further details on these implementation aspects, the reader is referred to our previous work [45].

In order to show the robustness of our method, we run the optimization on two test cases for two possible values of the minimum wall thickness $d_{\min }=0.1$ and $d_{\min }=0.04$. In both cases, the computational time required for running 359 iterations of the optimization algorithm is about 8 days on 30 processing units of an Intel(R) Xeon(R) CPU E5-2407 @ 2.4 GHz. We recall that only the finite element assemblies and linear system solutions are performed in parallel. The computational meshes were obtained with the isosurface discretization feature of Mmg by prescribing a maximum mesh element size $h_{\max }=0.04$ in the whole domain 


\begin{tabular}{|l|c|c|}
\hline Operation & Runtime (iteration 1) & Runtime (iteration 20) \\
\hline $\begin{array}{l}\text { Computation of the mesh connected } \\
\text { components and removal of isolated } \\
\text { fluid parts }\end{array}$ & $41 \mathrm{sec}$ & $21 \mathrm{sec}$ \\
\hline $\begin{array}{l}\text { Computation of the signed distance } \\
\text { function to } \Omega_{f}\end{array}$ & $31 \mathrm{sec}$ & $21 \mathrm{sec}$ \\
\hline $\begin{array}{l}\text { Computation of the signed distance } \\
\text { function to } \Omega_{f, \text { hot }}\end{array}$ & $14 \mathrm{sec}$ & $17 \mathrm{sec}$ \\
\hline $\begin{array}{l}\text { Computation of the signed distance } \\
\text { function to } \Omega_{f, \text { cold }}\end{array}$ & $22 \mathrm{sec}$ & $253 \mathrm{sec}$ \\
\hline $\begin{array}{l}\text { Solution of the state equations }(30 \text { pro- } \\
\text { cesses) }\end{array}$ & $344 \mathrm{sec}$ & $259 \mathrm{sec}$ \\
\hline $\begin{array}{l}\text { Computation of shape sensitivities }(30 \\
\text { processes) }\end{array}$ & $340 \mathrm{sec}$ & $8 \mathrm{sec}$ \\
\hline Advection of the solid-fluid interface & $11 \mathrm{sec}$ & $521 \mathrm{sec}$ \\
\hline $\begin{array}{l}\text { Isosurface discretization and remeshing } \\
\text { into a new computational mesh }\end{array}$ & $696 \mathrm{sec}$ & $19 \mathrm{~min}$ \\
\hline Total & $26 \mathrm{~min}$ & \\
\hline
\end{tabular}

TABLE 4. Main computational times for iterations 1 and 20 of the topology optimization algorithm in the solution of the $3 \mathrm{D}$ test case of section 5 with $d_{\min }=0.1$.

and a local maximum mesh element size $h_{\text {max,loc }}=0.015$ on the solid-fluid interface. This means that in the more "dangerous" configuration $d_{\min }=0.04$, the wall thickness prescribed between the two phases is of the order of only five times the local mesh size.

The initial mesh features about 3.8 million tetrahedra, 2.3 million of which lie inside the fluid domain. At every iteration, the mesh connected components associated with the hot and cold fluid channels are computed. Small isolated fluid components sometimes appear at topological changes during the optimization process. These are detected and removed at the beginning of every iteration.

The computational times observed for iterations 1 and 20 in the configuration $d_{\min }=0.1$ are reported in Table 4. Similar running times are observed in the case $d_{\min }=0.04$. During the optimization process, the mesh size decreases because the topology of the solid-fluid interface becomes simpler. For instance, in the case $d_{\min }=0.1$, the last mesh features of the order of 1.7 million tetrahedra including 686,000 in the fluid domain. As a result, the computational burden quickly decreases after a few iterations and remains constant after the 20th iteration till the end of the optimization process.

We note that the additional computational cost induced by the operations necessary to ensure the minimum distance constraint, which are carried out in parallel, is not substantial when compared to the total computational time of one iteration. Indeed, for the first iteration, the parallel assembly and solution of the variational problem (2.14) took about $70 \mathrm{sec}$ of the time allotted to compute shape sensitivities, $340 \mathrm{sec}$, cf. the 6 th row of Table 4.

\subsection{Three-dimensional numerical results}

The optimized distribution of the two fluid components $\Omega_{f \text {,hot }}$ and $\Omega_{f, \text { cold }}$, as well as several views of the fluid and the solid components $\Omega_{f}$ and $\Omega_{s}$ are represented on Figure 20 in the case $d_{\min }=0.1$, and on Figure 23 for $d_{\min }=0.04$. A few iterations of the optimization process are shown on Figs. 21 and 24 for these two respective configurations. For ease of visualisation in the configuration $d_{\min }=0.04$, the hot and cold phases are represented on two separate plots on Figure 26. These plots emphasize the fact that valid designs with 
a well-defined solid-fluid interface are available throughout the optimization process. We note that in both cases $d_{\min }=0.1$ and $d_{\text {min }}=0.04$, the optimization algorithm favors the appearance of transverse tubes in the hot phase during the first 200 iterations, before making them disappear in the last ones so that the pressure drop constraint be enforced more stringently.

The convergence histories for the objective and constraint functions are plotted on Figs. 22 and 25. We observe that in both configurations, the volume constraint on the hot fluid channel does not get saturated during the optimization process while it is the case for the cold fluid channel. The minimum distance constraint is initially violated but it becomes and remains satisfied after about 30 iterations, although dramatic topological and shape changes still occur thenceforth.

The optimized designs are rather unconventional, since they do not feature a network of pipes as one would have intuitively expected, but rather an arrangement of fluid surfaces. We note that the final distribution of fluid are naturally free from nonphysical three-dimensional solid islands, a feature which not enforced by explicit means in our computation.

\section{Conclusion and Perspectives}

This article proposed a framework for the shape and topology optimization of two models of heat exchangers, based on the boundary variation method of Hadamard. In a first part, a simplified cross-flow 2D model was investigated, demonstrating the ability of our numerical method to generate a large variety of optimized designs associated to different physical parameters and initial guesses. Our results pointed out that, according to the initial topology, different optimal shapes may be satisfactory candidates for one single configuration of the heat exchanger design problem.

A second part considered more realistic two-network heat exchangers models, for which our framework conveniently allowed to enforce a non-penetration constraint between both types (hot and cold) of channels. The efficiency of our approach was demonstrated on two 2D cases and on a moderately large-scale 3D case. Although the test cases considered in this paper remains somehow academic, our results further highlight the capabilities of topology optimization to generate unconventional designs which could be of interest in the prospect of longer-term industrial applications.

One natural lead for future work would be to deal with other physical models than those of sections 3.1 and 4.1, in order to handle, for instance, turbulent situations. Let us mention, however, that several difficulties are still to be expected regarding the applications of the present shape optimization methods to such realistic industrial applications featuring large Reynolds and Péclet numbers, which are typically of the order of 30,000 or beyond. Indeed, when the Reynolds number is large, the assumption of stationary velocity and pressure fields may break down because stationary solutions become asymptotically unstable. The timedependent solution rather tends to evolve to an attractor [88]. This could motivate the need for considering turbulence models which enable to approximate the mean stationary fields and to capture boundary layers by using sufficiently fine meshes. In a second extent, one could consider time-dependent models in such situations, although these are known to induce substantial additional computational costs due to the need for computing adjoint solutions backward in time, see [9] for one instance of such a situation.

Finally, the current shapes of industrial heat exchanger suggest that optimized designs could be realized by multi-scale patterns, featuring many small details such as fins or tubes, repeated in a periodic fashion. Such designs would be quite difficult to mesh explicitly, and so to capture with the method of Hadamard. However, it is tempting to consider the use of homogenization approaches in this context, which are specifically devised to generate multi-scale geometries.

The recent works $[75,46,8]$ offer interesting perspectives regarding the automatic generation of optimized microstructures from homogenized models in the context of linear elasticity. In [41], a few preliminary theoretical contributions are outlined towards the application of such methods in the context of fluid systems. In complement, let us mention the recent publication [32] where promising results are discussed regarding the dehomogenization of fluid flow channel networks based on the Darcy model.

Acknowledgements. This work is financed by the Association Nationale de la Recherche et de la Technologie (ANRT), grant number CIFRE 2017/0024, and by project ANR-18-CE40-0013 SHAPO from the French Agence Nationale de la Recherche (ANR). F. F. is partially funded by a CIFRE grant by SAFRAN, 

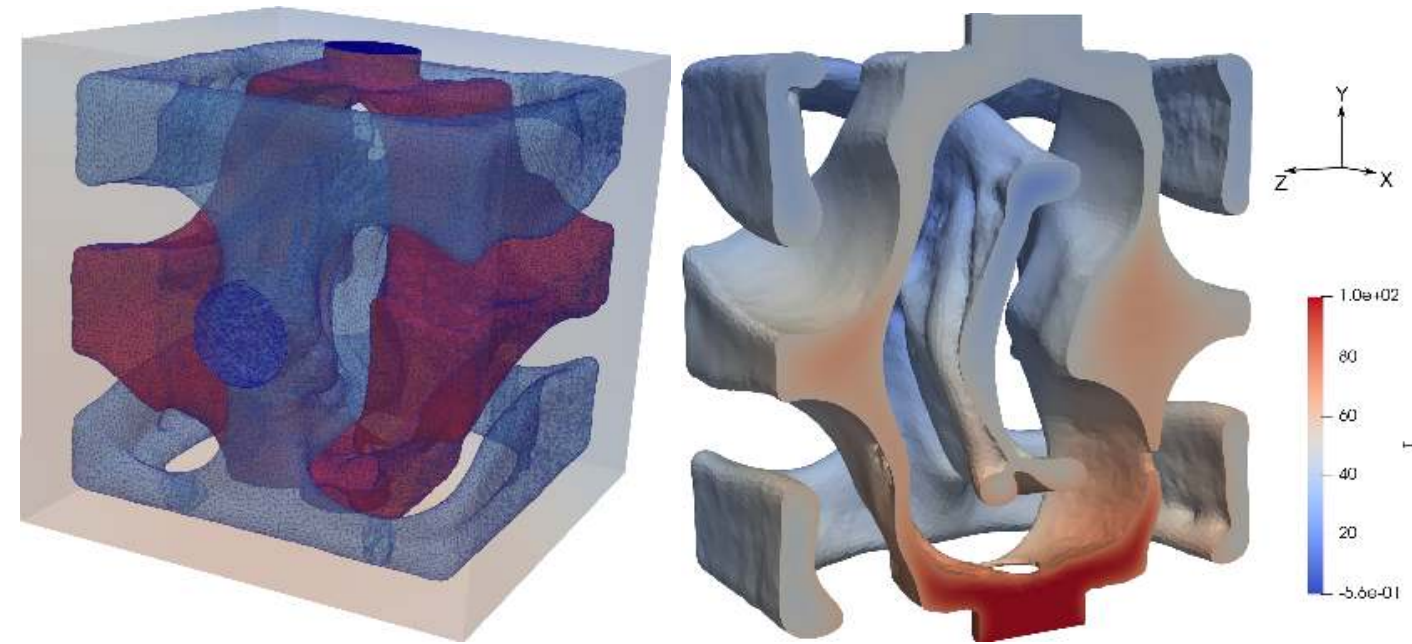

(A) Optimized distribution of the cold and hot fluid (в) Sectional view of the topologically optimized fluid docomponents, respectively colored in blue and red.

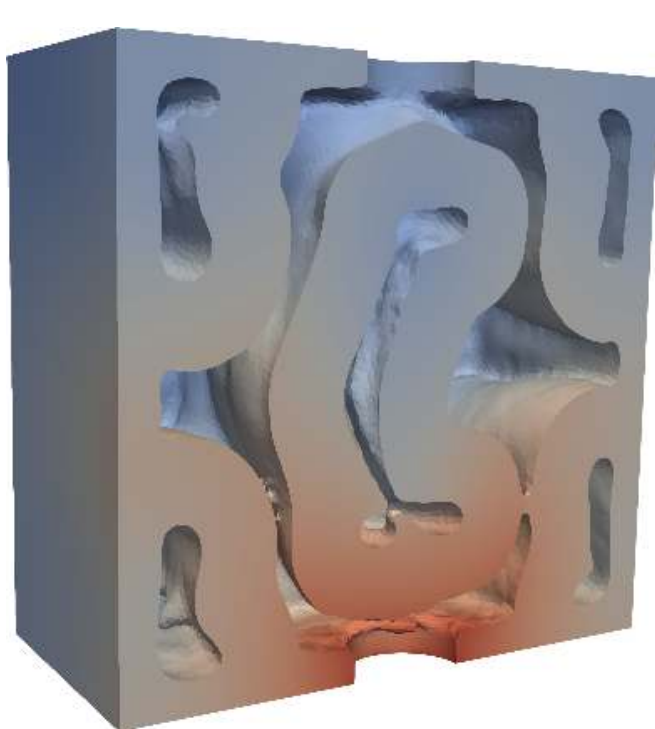

(c) Sectional view of the topologically optimized solid domain. The color corresponds to the temperature profile. Observe the wall thickness greater than 0.1 prescribed between the two fluid components.

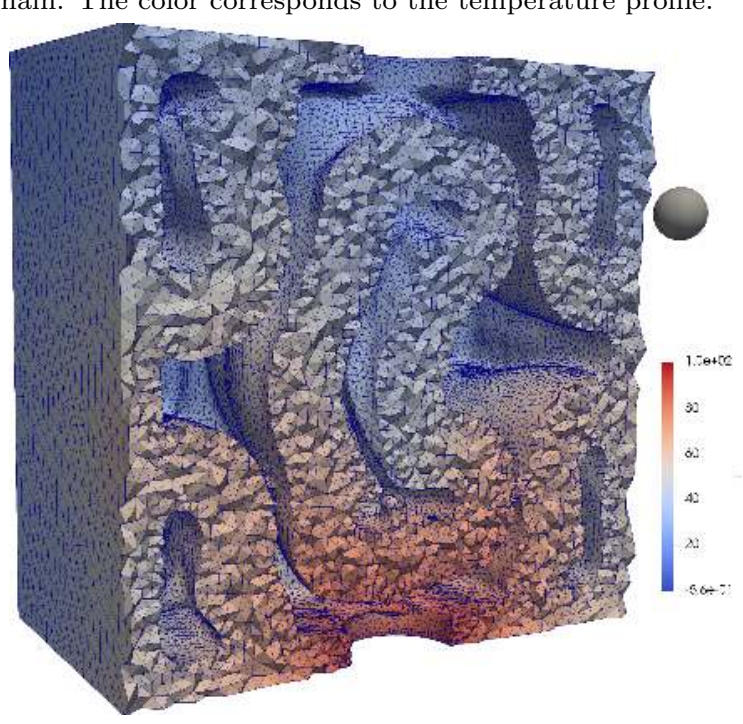

(D) Sectional view of the topologically optimized solid domain with tetrahedral mesh elements made visible. Observe that both fluid components are separated from only 9 to 10 mesh elements. The diameter of the sphere on the right-hand side is equal to the prescribed minimum distance constraint $d_{\min }=0.1$.

Figure 20. Final design for the three-dimensional heat exchanger test case of section 5.

the support of which is kindly acknowledged. We are grateful to the engineers of Safran Aeroboosters whose collaboration led us to the design of the test case tackled in section 3 .

\section{REFERENCES}

[1] N. Aage, E. Andreassen, B. S. Lazarov, and O. Sigmund, Giga-voxel computational morphogenesis for structural design, Nature, 550 (2017), p. 84

[2] J. Alexandersen and C. S. Andreasen, A review of topology optimisation for fluid-based problems, Fluids, 5 (2020), p. 29.

[3] J. Alexandersen, O. Sigmund, And N. Aage, Large scale three-dimensional topology optimisation of heat sinks cooled by natural convection, International Journal of Heat and Mass Transfer, 100 (2016), pp. 876-891. 

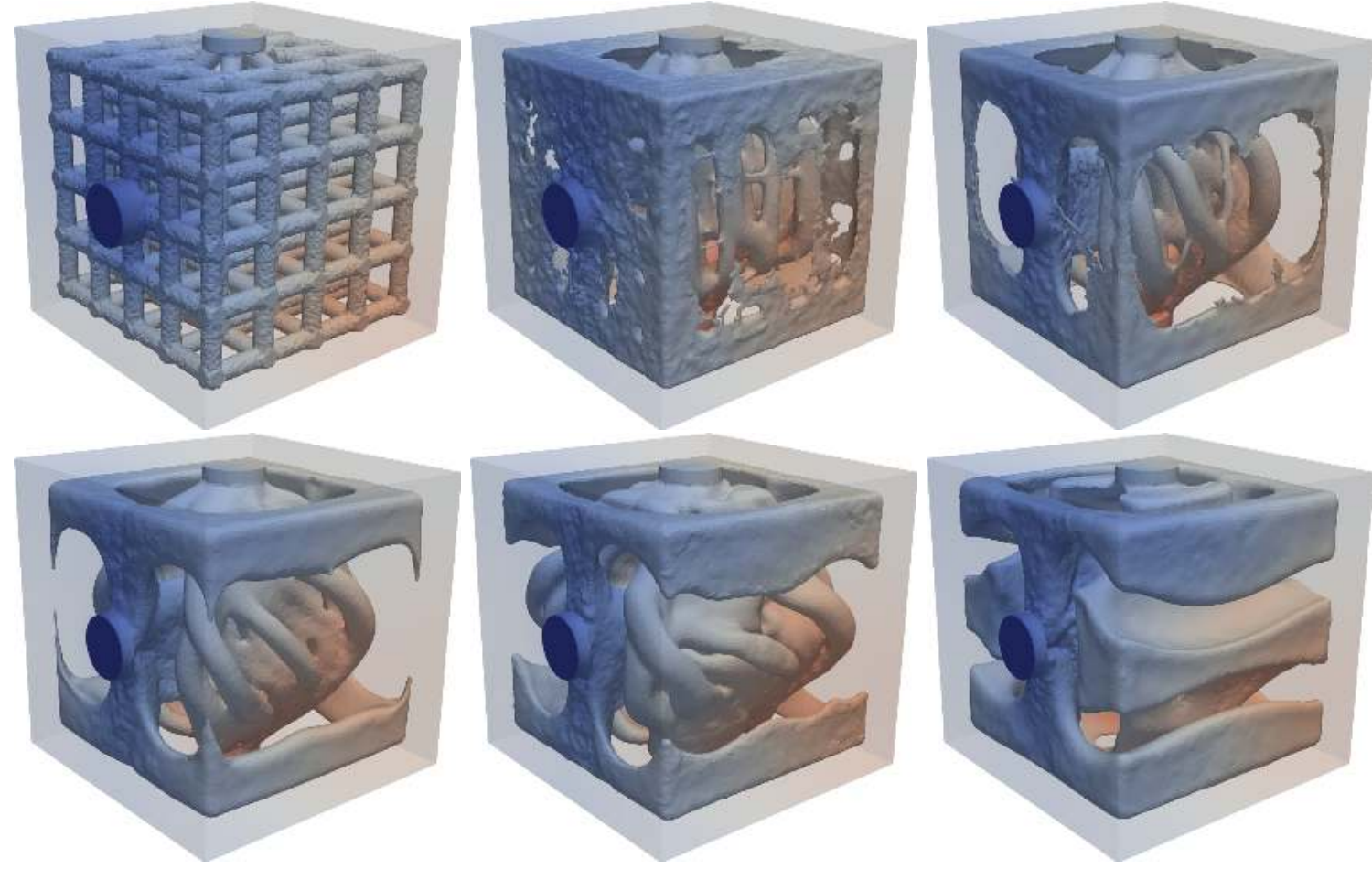

FiguRE 21. Iterations $0,25,50,110,180$, and 360 of the shape optimization of the solid-fluid interface in the three-dimensional heat exchanger test case of section 5 with $d_{\min }=0.1$. The colors correspond to the magnitude of the temperature field. Both fluid components remain well-separated throughout the optimization process.

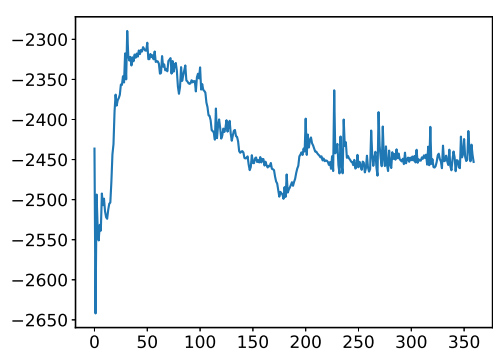

(A) Objective function (opposite of the heat exchanged).

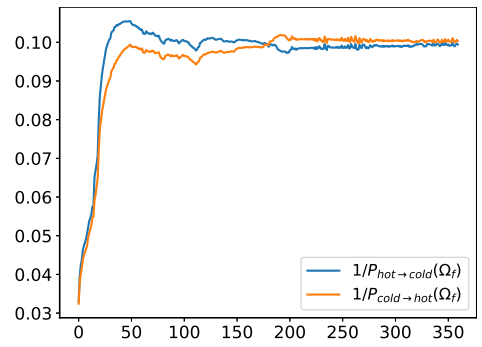

(C) Averaged distance between the two phases.

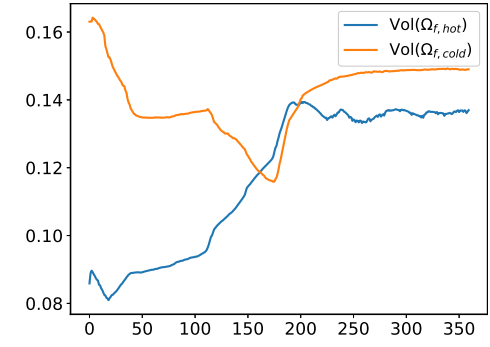

(B) Volume fractions. Final values: $\operatorname{Vol}\left(\Omega_{f, h o t}\right)=0.137$, $\operatorname{Vol}\left(\Omega_{f, \text { cold }}\right)=0.149$.

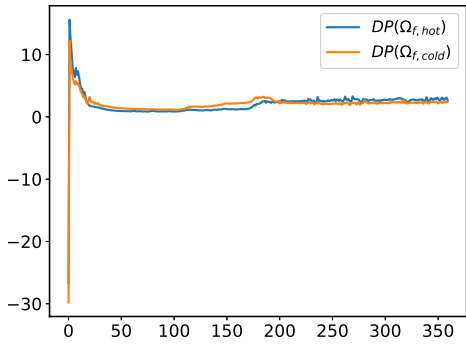

(D) Pressure drop. Final values: $\mathrm{DP}\left(\Omega_{f, \text { hot }}\right)=3.16$, $\mathrm{DP}\left(\Omega_{f, \text { cold }}\right)=2.87$.

FIgURE 22. Convergence histories of the 3D heat exchanger test case of section 5 with $d_{\min }=0.1$. 

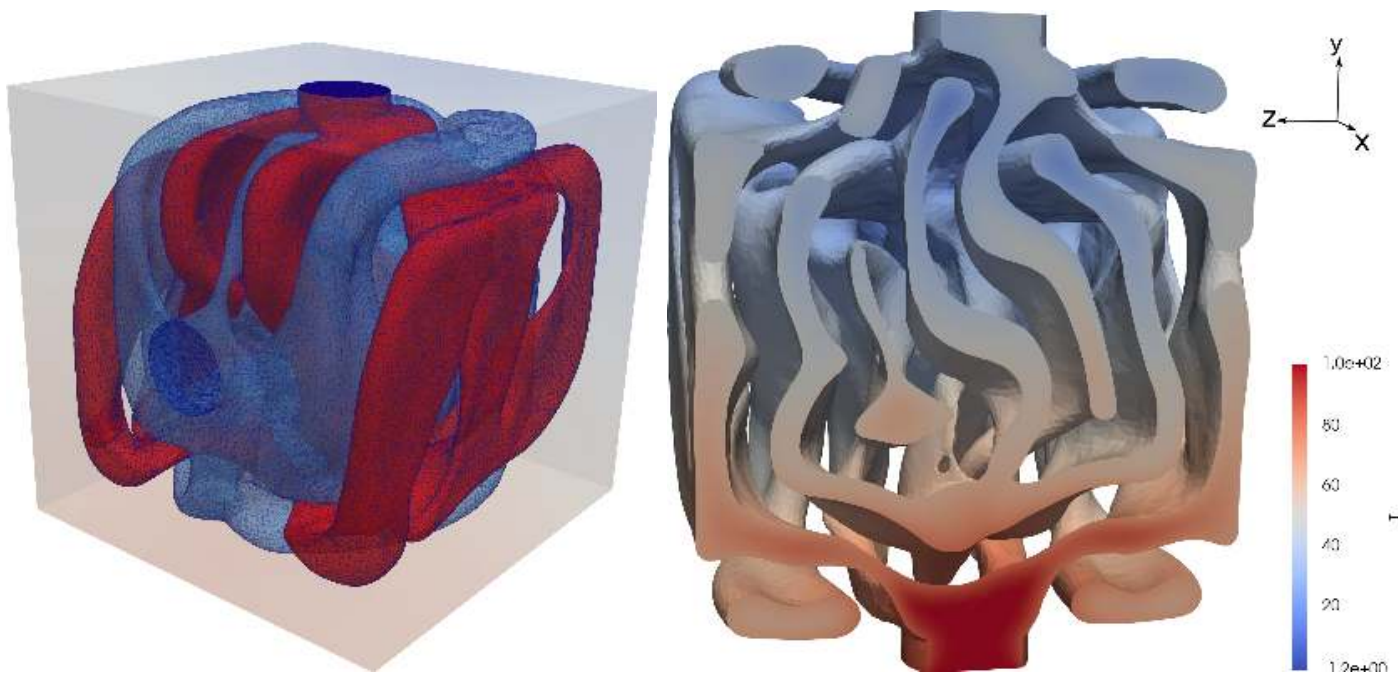

(A) Optimized distribution of the cold and hot fluid components, respectively colored in blue and red.
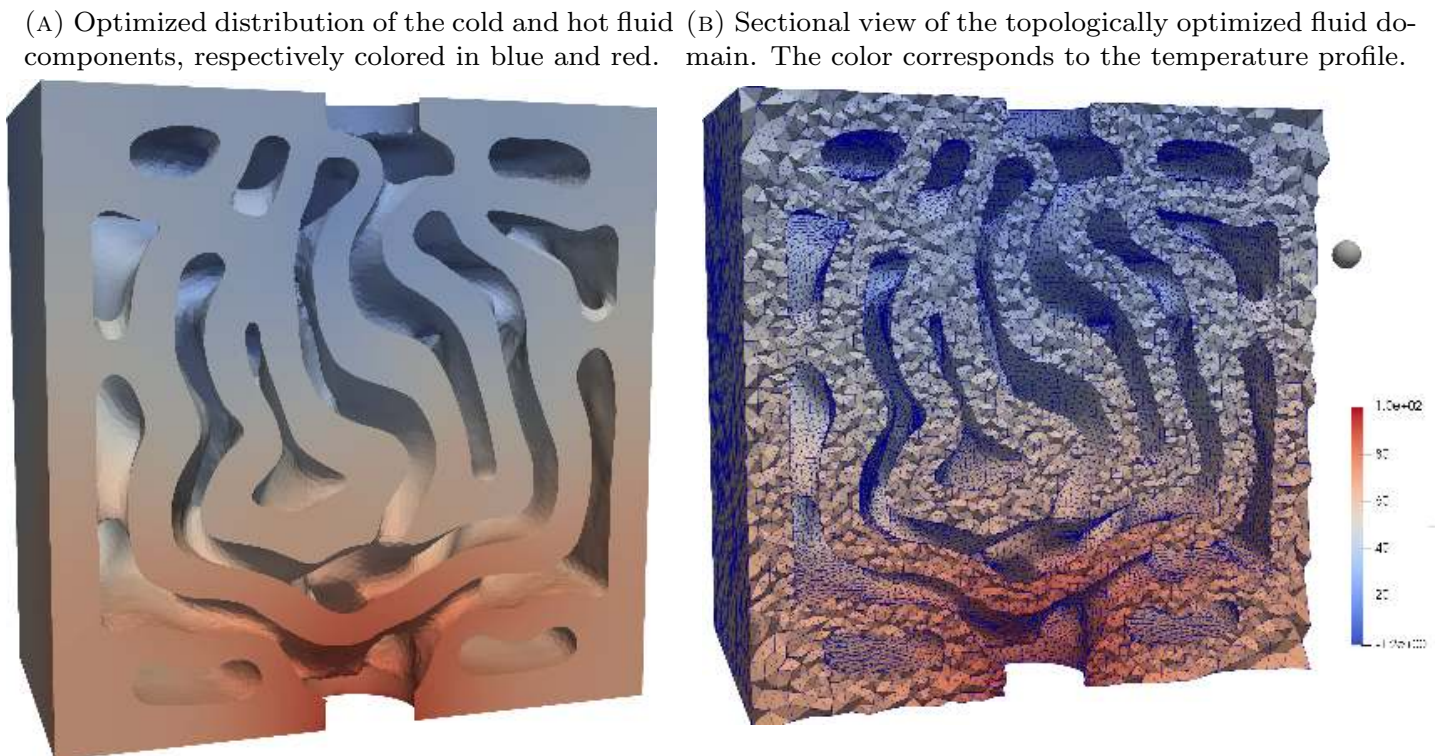

(c) Sectional view of the topologically optimized solid domain. The color corresponds to the temperature profile. Observe the wall thickness greater than 0.04 prescribed between the two fluid components.

(D) Sectional view of the topologically optimized solid domain with tetrahedral mesh elements made visible. The diameter of the sphere on the right-hand side is equal to the prescribed minimum distance constraint $d_{\min }=0.04$.

Figure 23. Final design for the three-dimensional heat exchanger test case of section 5 with $d_{\min }=0.04$.

[4] G. Allaire, C. Dapogny, G. Delgado, and G. Michailidis, Multi-phase structural optimization via a level set method, ESAIM: Control, Optimisation and Calculus of Variations, 20 (2014), pp. 576-611.

[5] G. Allaire, C. Dapogny, And P. Frey, Topology and geometry optimization of elastic structures by exact deformation of simplicial mesh, Comptes Rendus Mathematique, 349 (2011), pp. 999-1003.

[6] — Shape optimization with a level set based mesh evolution method, Computer Methods in Applied Mechanics and Engineering, 282 (2014), pp. 22-53.

[7] G. Allaire, C. Dapogny, and F. Jouve, Shape and topology optimization, to appear in Handbook of Numerical Analysis, Vol. 20, Hal preprint https://hal.archives-ouvertes.fr/hal-02496063/, (2020).

[8] G. Allaire, P. Geoffroy Donders, and O. Pantz, Topology optimization of modulated and oriented periodic microstructures by the homogenization method, Computers \& Mathematics with Applications, 78 (2019), pp. 2197-2229.

[9] G. Allaire AND L. JaKabcin, Taking into account thermal residual stresses in topology optimization of structures built by additive manufacturing, Mathematical Models and Methods in Applied Sciences, 28 (2018), pp. 2313-2366. 

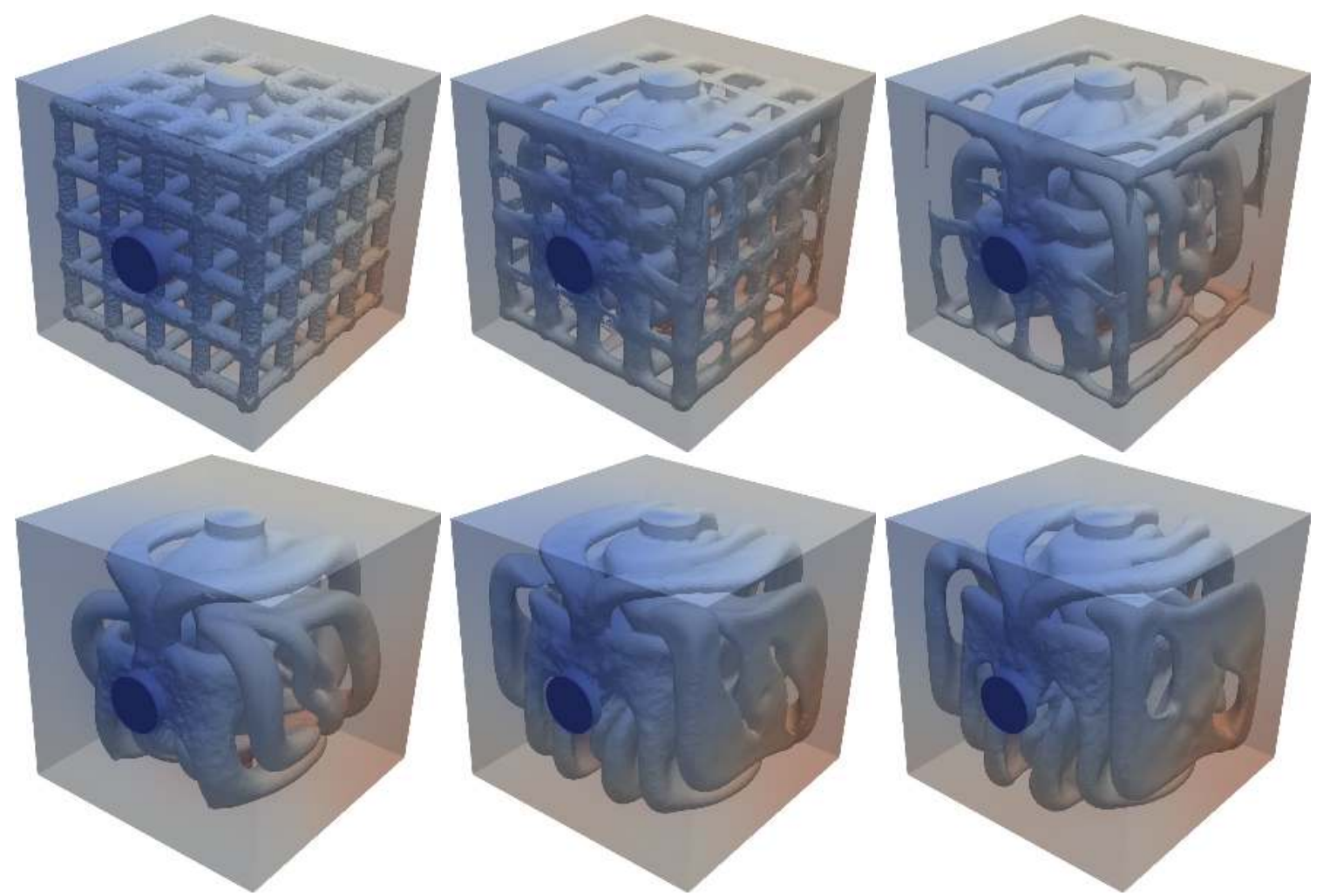

FIGURE 24. Iterations $0,25,50,130,200$ and 500 of the shape optimization of the solid-fluid interface in the three-dimensional heat exchanger test case of section 5 with $d_{\min }=0.04$. The colors correspond to the magnitude of the temperature field. Both fluid components remain wellseparated throughout the optimization process.

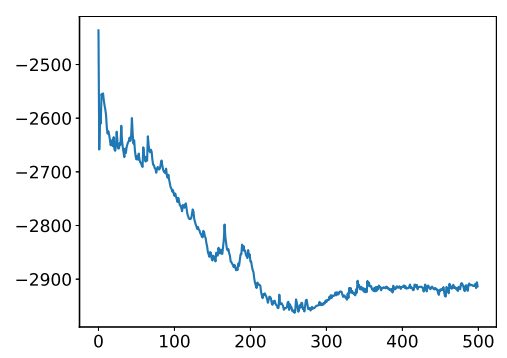

(A) Objective function (opposite of the heat exchanged).

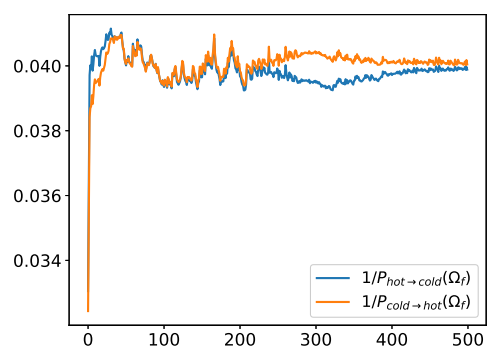

(C) Averaged distance between the two phases.

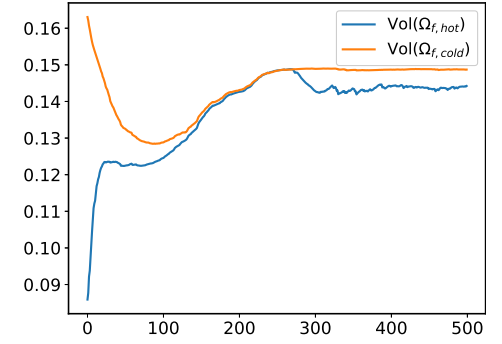

(в) Volume fractions. Final values: $\operatorname{Vol}\left(\Omega_{f}\right.$, hot $)=0.144$, $\operatorname{Vol}\left(\Omega_{f, \text { cold }}\right)=0.149$.

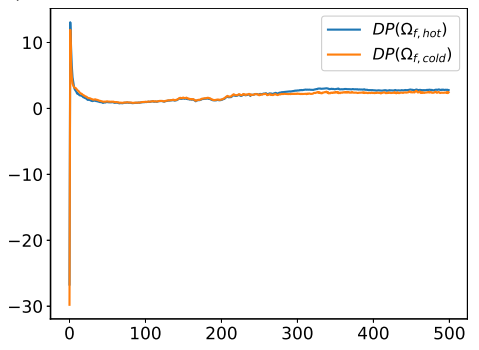

(D) Pressure drop. Final values: $\operatorname{DP}\left(\Omega_{f, \text { hot }}\right)=3.26$, $\operatorname{DP}\left(\Omega_{f, \text { cold }}\right)=2.92$.

Figure 25. Convergence histories of the 3D heat exchanger test case of section 5 with $d_{\min }=0.04$. 


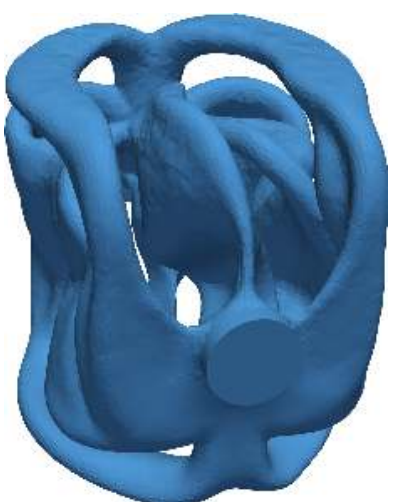

(A) Cold phase

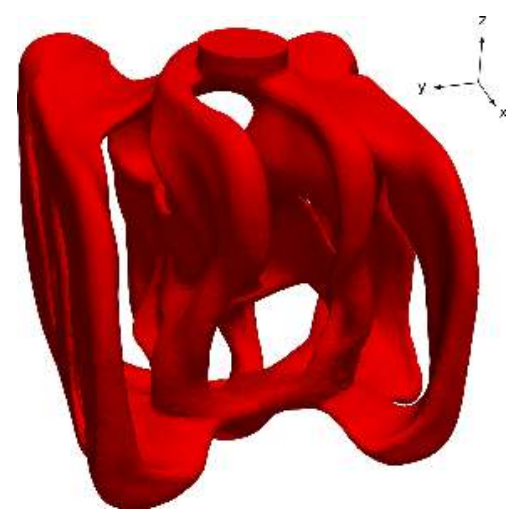

(B) Hot phase

Figure 26. Separate plots of the topologically optimized cold and hot fluid phases in the configuration $d_{\min }=0.04$.

[10] G. Allaire, F. Jouve, And G. Michailidis, Thickness control in structural optimization via a level set method, Structural and Multidisciplinary Optimization, 53 (2016), pp. 1349-1382.

[11] G. Allaire, F. Jouve, And A. Toader, A level-set method for shape optimization, Comptes Rendus Mathematique, 334 (2002), pp. 1125-1130.

[12] G. Allaire, F. Jouve, And A.-M. Toader, Structural optimization using sensitivity analysis and a level-set method, Journal of Computational Physics, 194 (2004), pp. 363-393.

[13] O. Amir, N. Aage, and B. S. Lazarov, On multigrid-CG for efficient topology optimization, Structural and Multidisciplinary Optimization, 49 (2014), pp. 815-829.

[14] M. Awais AND A. A. BhUiYAn, Heat and mass transfer for compact heat exchanger (CHXs) design: A state-of-the-art review, International Journal of Heat and Mass Transfer, 127 (2018), pp. 359-380.

[15] S. Balay, S. Abhyankar, M. F. Adams, J. Brown, P. Brune, K. Buschelman, L. Dalcin, A. Dener, V. Eijkhout, W. D. Gropp, D. Karpeyev, D. Kaushik, M. G. Knepley, D. A. May, L. C. McInnes, R. T. Mills, T. Munson, K. Rupp, P. Sanan, B. F. Smith, S. Zampini, H. Zhang, and H. Zhang, PETSc users manual, Tech. Rep. ANL-95/11 - Revision 3.11, Argonne National Laboratory, 2019.

[16] — PETSC Web page. https://www.mcs.anl.gov/petsc, 2019.

[17] R. Becker, V. Heuveline, and R. Rannacher, An optimal control approach to adaptivity in computational fluid mechanics, International journal for numerical methods in fluids, 40 (2002), pp. 105-120.

[18] M. M. A. Bhutta, N. Hayat, M. H. Bashir, A. R. Khan, K. N. Ahmad, and S. Khan, CFD applications in various heat exchangers design: A review, Applied Thermal Engineering, 32 (2012), pp. 1-12.

[19] T. Borrvall and J. Petersson, Large-scale topology optimization in 3D using parallel computing, Computer Methods in Applied Mechanics and Engineering, 190 (2001), pp. 6201-6229.

[20] - Topology optimization of fluids in Stokes flow, International Journal for Numerical Methods in Fluids, 41 (2003), pp. $77-107$.

[21] M. BURGER, A framework for the construction of level set methods for shape optimization and reconstruction, Interfaces and Free boundaries, 5 (2003), pp. 301-329.

[22] E. CAPEY, Alleviation of thermal stresses in aircraft structures. ministry of aviation, aeronautical research council. CP \#819, 1965.

[23] J. Chetboun, Conception de formes aérodynamiques en présence d'écoulements décollés : contrôle et optimisation, PhD thesis, École Polytechnique, 2010.

[24] L. Cirrottola And A. Froehly, Parallel unstructured mesh adaptation using iterative remeshing and repartitioning, Research Report 9307, INRIA Bordeaux, équipe CARDAMOM, 2019.

[25] P. Coffin And K. Maute, Level set topology optimization of cooling and heating devices using a simplified convection model, Structural and Multidisciplinary Optimization, 53 (2016), pp. 985-1003.

[26] C. DApogny, C. Dobrzynski, And P. FReY, Three-dimensional adaptive domain remeshing, implicit domain meshing, and applications to free and moving boundary problems, Journal of Computational Physics, 262 (2014), pp. 358-378.

[27] C. Dapogny, C. Dobrzynski, P. Frey, and A. Froelhy, Mmg. https://www.mmgtools.org, 2019.

[28] C. Dapogny And P. Frey, Computation of the signed distance function to a discrete contour on adapted triangulation, Calcolo, 49 (2012), pp. 193-219.

[29] T. Dвоuк, A review about the engineering design of optimal heat transfer systems using topology optimization, Applied Thermal Engineering, 112 (2017), pp. 841-854.

[30] F. DE GouRnAY, Velocity extension for the level-set method and multiple eigenvalues in shape optimization, SIAM Journal on Control and Optimization, 45 (2006), pp. 343-367. 
[31] E. M. DEDE, Multiphysics topology optimization of heat transfer and fluid flow systems, in proceedings of the COMSOL Users Conference, 2009.

[32] E. M. Dede, Y. Zhou, And T. Nomura, Inverse design of microchannel fluid flow networks using Turing pattern dehomogenization, Structural and Multidisciplinary Optimization, (2020).

[33] M. C. Delfour And J.-P. ZolÉsio, Shapes and geometries: metrics, analysis, differential calculus, and optimization, SIAM, 2011.

[34] C. Dilgen, S. Dilgen, D. Fuhrman, O. Sigmund, and B. Lazarov, Topology optimization of turbulent flows, Computer Methods in Applied Mechanics and Engineering, 331 (2018), pp. 363-393.

[35] S. B. Dilgen, C. B. Dilgen, D. R. Fuhrman, O. Sigmund, and B. S. Lazarov, Density based topology optimization of turbulent flow heat transfer systems, Structural and Multidisciplinary Optimization, 57 (2018), pp. 1905-1918.

[36] Q. V. Dinh, G. Rogé, C. Sevin, and B. Stoufflet, Shape optimization in computational fluid dynamics, Revue Européenne des Éléments Finis. European Journal of Finite Elements, 5 (1996), pp. 569-594.

[37] T. Dupont, M. F. Wheeler, et Al., A Galerkin procedure for approximating the flux on the boundary for elliptic and parabolic boundary value problems, Revue française d'automatique, informatique, recherche opérationnelle. Analyse numérique, 8 (1974), pp. 47-59.

[38] M. C. Duta, S. Shahpar, And M. B. Giles, Turbomachinery design optimization using automatic differentiated adjoint code, in ASME Turbo Expo 2007: Power for Land, Sea, and Air, American Society of Mechanical Engineers, 2007, pp. $1435-1444$.

[39] B. Epstein, A. Jameson, S. Peigin, D. Roman, N. Harrison, and J. Vassberg, Comparative study of three-dimensional wing drag minimization by different optimization techniques, Journal of Aircraft, 46 (2009), pp. 526-541.

[40] F. Feppon, Shape and topology optimization of multiphysics systems, PhD thesis, École Polytechnique, 2019.

[41] — High order homogenization of the Stokes system in a periodic porous medium. Preprint, Jun 2020.

[42] F. Feppon, G. Allaire, F. Bordeu, J. Cortial, and C. Dapogny, Shape optimization of a coupled thermal fluidstructure problem in a level set mesh evolution framework, SeMA, 76 (2019), pp. 413-458.

[43] F. Feppon, G. Allaire, And C. Dapogny, Null space gradient flows for constrained optimization with applications to shape optimization, ESAIM: COCV, Forthcoming article, (2019).

[44] F. Feppon, G. Allaire, and C. Dapogny, A variational formulation for computing shape derivatives of geometric constraints along rays, ESAIM: M2AN, 54 (2020), pp. 181-228.

[45] F. Feppon, G. Allaire, C. Dapogny, And P. Jolivet, Topology optimization of thermal fluid-structure systems using body-fitted meshes and parallel computing, Journal of Computational Physics, 417 (2020), p. 109574.

[46] P. Geoffroy Donders, Homogenization method for topology optimization of structures built with lattice materials, PhD thesis, Université Paris-Saclay, 2018.

[47] M. Giacomini, O. Pantz, and K. Trabelsi, Volumetric expressions of the shape gradient of the compliance in structural shape optimization, arXiv:1701.05762, (2017).

[48] J. H. K. Haertel, Design of Thermal Systems Using Topology Optimization, PhD thesis, Technical University of Denmark, 2018.

[49] S. Harries, C. Abt, And M. Brenner, Upfront CAD-Parametric modeling techniques for shape optimization, in Advances in Evolutionary and Deterministic Methods for Design, Optimization and Control in Engineering and Sciences, vol. 48, Springer, 2019, pp. 191-211.

[50] F. Hecht, New development in FreeFem++, Journal of Numerical Mathematics, 20 (2012), pp. 251-265.

[51] A. Henrot and M. Pierre, Shape variation and optimization, vol. 28 of EMS Tracts in Mathematics, European Mathematical Society (EMS), Zürich, 2018.

[52] R. Hiptmair, A. Paganini, and S. Sargheini, Comparison of approximate shape gradients, BIT Numerical Mathematics, 55 (2015), pp. 459-485.

[53] G. P. Huang, D. Doman, M. Oppenheimer, A. Tipton, and D. Sigthorsson, Topology optimization of a fuel thermal management system, in AIAA Aviation 2019 Forum, 2019, p. 3471.

[54] L. C. Hoghoj, D. R. Norhave, J. Alexandersen, O. Sigmund, and C. S. Andreasen, Topology Optimization of TwoFluid Heat Exchangers, arXiv:2007.01759, (2020).

[55] F. P. Incropera, D. P. DeWitt, T. L. Bergman, and A. S. Lavine, Foundations of Heat Transfer, 6th Edition International Student Version, Wiley, Jun 2012.

[56] S. Kambampati, C. Jauregui, K. Museth, and H. A. Kim, Fast level set topology optimization using a hierarchical data structure, in 2018 Multidisciplinary Analysis and Optimization Conference, 2018, p. 3881.

[57] H. Kobayashi, K. Yaji, S. Yamasaki, And K. Fujita, Freeform winglet design of fin-and-tube heat exchangers guided by topology optimization, Applied Thermal Engineering, 161 (2019), p. 114020.

[58] H. Kobayashi, K. Yaji, S. Yamasaki, and K. Fujita, Topology design of two-fluid heat exchange, arXiv:2005.08870, (2020).

[59] A. A. Koga, E. C. C. Lopes, H. F. V. Nova, C. R. De Lima, and E. C. N. Silva, Development of heat sink device by using topology optimization, International Journal of Heat and Mass Transfer, 64 (2013), pp. 759-772.

[60] M. Laurent, R. Estevez, D. Fabrègue, and E. Ayax, Thermomechanical fatigue life prediction of 316L compact heat exchanger, Engineering Failure Analysis, 68 (2016), pp. 138-149.

[61] J. LiU, L. LI, AND Y. MA, Uniform thickness control without pre-specifying the length scale target under the level set topology optimization framework, Advances in Engineering Software, 115 (2018), pp. 204-216. 
[62] J. LiU And Y. MA, A survey of manufacturing oriented topology optimization methods, Advances in Engineering Software, 100 (2016), pp. 161-175.

[63] C. Mantegazza and A. C. Mennucci, Hamilton-Jacobi Equations and Distance Functions on Riemannian Manifolds, Applied Mathematics \& Optimization, 47 (2003).

[64] G. Marck, M. Nemer, And J.-L. Harion, Topology optimization of heat and mass transfer problems: Laminar flow, Numerical Heat Transfer, Part B: Fundamentals, 63 (2013), pp. 508-539.

[65] G. Marck And Y. PRIVAT, On some shape and topology optimization problems in conductive and convective heat transfers, in OPTI 2014, An International Conference on Engineering and Applied Sciences Optimization, M. Papadrakakis, M. Karlaftis, and N. Lagaros, eds., June 2014, pp. 1640-1657.

[66] J. Martínez-Frutos and D. Herrero-PÉrez, GPU acceleration for evolutionary topology optimization of continuum structures using isosurfaces, Computers \& Structures, 182 (2017), pp. 119-136.

[67] L. A. M. Mello, E. De Sturler, G. H. Paulino, and E. C. N. Silva, Recycling Krylov subspaces for efficient large-scale electrical impedance tomography, Computer Methods in Applied Mechanics and Engineering, 199 (2010), pp. 3101-3110.

[68] G. MichaiLIDIS, Manufacturing Constraints and Multi-Phase Shape and Topology Optimization via a Level-Set Method, PhD thesis, École Polytechnique, 2014.

[69] M. H. Mohammadi, H. R. Abbasi, A. Yavarinasab, and H. Pourrahmani, Thermal optimization of shell and tube heat exchanger using porous baffles, Applied Thermal Engineering, 170 (2020), p. 115005.

[70] D. J. Munk, T. Kipouros, G. A. Vio, G. T. Parks, and G. P. Steven, On the effect of fluid-structure interactions and choice of algorithm in multi-physics topology optimisation, Finite Elements in Analysis and Design, 145 (2018), pp. 32-54.

[71] F. Murat And J. Simon, Sur le contrôle par un domaine géométrique, Publication du Laboratoire d'Analyse Numérique de l'Université Pierre et Marie Curie, (1976).

[72] S. Osher And R. Fedkiw, Level set methods and dynamic implicit surfaces, vol. 153 of Applied Mathematical Sciences, Springer-Verlag, New York, 2003.

[73] S. Osher And J. A. Sethian, Fronts propagating with curvature-dependent speed: algorithms based on Hamilton-Jacobi formulations, Journal of Computational Physics, 79 (1988), pp. 12-49.

[74] S. J. Osher And F. SAntosa, Level set methods for optimization problems involving geometry and constraints. I. Frequencies of a two-density inhomogeneous drum, Journal of Computational Physics, 171 (2001), pp. $272-288$.

[75] O. Pantz and K. Trabelsi, A post-treatment of the homogenization method for shape optimization, SIAM Journal on Control and Optimization, 47 (2008), pp. 1380-1398.

[76] P. PAPazoglou, Topology optimization of heat exchangers, Master's thesis, TU Delft, 2015.

[77] P.-O. Persson, Mesh generation for implicit geometries, PhD thesis, Massachusetts Institute of Technology, 2005.

[78] M. Pietropaoli, F. Montomoli, and A. Gaymann, Three-dimensional fluid topology optimization for heat transfer, Structural and Multidisciplinary Optimization, 59 (2019), pp. 801-812.

[79] A. ReBeI, Développement de méthode d'optimisation topologique adaptée aux écoulements en régime turbulent, application au cas des échangeurs de chaleur, PhD thesis, Université Paris Sciences et Lettres, 2019.

[80] D. Saltzman, M. Bichnevicius, S. Lynch, T. W. Simpson, E. W. Reutzel, C. Dickman, and R. Martukanitz, Design and evaluation of an additively manufactured aircraft heat exchanger, Applied Thermal Engineering, 138 (2018), pp. 254-263.

[81] K. R. Saviers, R. Ranjan, And R. Mahmoudi, Design and validation of topology optimized heat exchangers, in AIAA Scitech 2019 Forum, 2019, p. 1465.

[82] J. A. Sethian, Level set methods and fast marching methods: evolving interfaces in computational geometry, fluid mechanics, computer vision, and materials science, vol. 3, Cambridge University Press, 1999.

[83] J. A. Sethian And A. Wiegmann, Structural boundary design via level set and immersed interface methods, Journal of Computational Physics, 163 (2000), pp. 489-528.

[84] R. K. Shah and D. P. SeKulic, Fundamentals of heat exchanger design, John Wiley \& Sons, 2003.

[85] J. Sobieszczanski-Sobieski And R. T. HAftKA, Multidisciplinary aerospace design optimization: survey of recent developments, Structural Optimization, 14 (1997), pp. 1-23.

[86] J. Sokolowski AND J.-P. ZolÉSIO, Introduction to shape optimization, vol. 16 of Springer Series in Computational Mathematics, Springer-Verlag, Berlin, 1992.

[87] R. Tawk, B. Ghannam, And M. Nemer, Topology optimization of heat and mass transfer problems in two fluids-one solid domains, Numerical Heat Transfer, Part B: Fundamentals, 76 (2019), pp. 130-151.

[88] R. Temam, Navier-Stokes equations: Theory and numerical analysis, vol. 45, North-Holland Publishing Company, 1977.

[89] M. Y. WAng, X. WAng, And D. Guo, A level set method for structural topology optimization, Computer Methods in Applied Mechanics and Engineering, 192 (2003), pp. 227-246.

[90] G. H. Yoon, Topological layout design of electro-fluid-thermal-compliant actuator, Computer Methods in Applied Mechanics and Engineering, 209-212 (2012), pp. 28-44.

[91] - Stress-based topology optimization method for steady-state fluid-structure interaction problems, Computer Methods in Applied Mechanics and Engineering, 278 (2014), pp. 499-523. 